\title{
Characterization of Mg-based Bimetal Treatment of Insensitive Munition 2,4-dinitroanisole
}

Emese Hadnagy

University of New Haven, ehadnagy@newhaven.edu

Andrew Mai

Stevens Institute of Technology

Benjamin Smolinski

United States Army

Washington Braida

Stevens Institute of Technology

Agamemnon Koutsospyros

University of New Haven, akoutsospyros@newhaven.edu

Follow this and additional works at: https://digitalcommons.newhaven.edu/civilengineeringfacpubs

Part of the Civil Engineering Commons

\section{Publisher Citation}

Hadnagy, E., Mai, A., Smolinski, B., Braida, W., \& Koutsospyros, A. (2018). Characterization of Mg-based bimetal treatment of insensitive munition 2,4-dinitroanisole. Environmental Science and Pollution Research, 1-14.

\section{Comments}

This is the authors' accepted manuscript of the article published in Environmental Science and Pollution Research. The article of record can be found at http://dx.doi.org/10.1007/s11356-018-2493-1. 
*Corresponding Author. Email address: EHadnagy@newhaven.edu ${ }^{1}$ Department of Civil and Environmental Engineering, University of New Haven ${ }^{2}$ Department of Civil, Environmental, and Ocean Engineering, Stevens Institute of Technology ${ }^{3}$ RDECOM-ARDEC

Distribution A: Approved for Public Release; Distribution is Unlimited 


\section{$8 \quad$ Abstract}

9 The manufacturing of insensitive munition 2,4-dinitroanisole (DNAN) generates waste streams

10 that require treatment. DNAN has been treated previously with zero-valent iron (ZVI) and Fe-

11 based bimetals. Use of Mg-based bimetals offers certain advantages including potential higher

12 reactivity and relative insensitivity to $\mathrm{pH}$ conditions. This work reports preliminary findings of

13 DNAN degradation by three $\mathrm{Mg}$-based bimetals: $\mathrm{Mg} / \mathrm{Cu}, \mathrm{Mg} / \mathrm{Ni}$, and $\mathrm{Mg} / \mathrm{Zn}$. Treatment of

14 DNAN by all three bimetals is highly effective in aqueous solutions ( $>89 \%$ removal) and

15 wastewater ( $>91 \%$ removal) in comparison to treatment solely with zero-valent magnesium

16 (ZVMg; 35\% removal). Investigation of reaction byproducts supports a partial degradation

17 pathway involving reduction of the ortho or para nitro- to amino- group, leading to 2-amino-4-

18 nitroanisole (2-ANAN) and 4-amino-2-nitroanisole (4-ANAN). Further reduction of the second

19 nitro group leads to 2, 4-diaminoanisole (DAAN). These byproducts are detected in small

20 quantities in the aqueous phase. Carbon mass balance analysis suggests near complete closure

$21(91 \%)$ with $12.4 \%$ and $78.4 \%$ of the total organic carbon (TOC) distributed in the aqueous and

22 mineral bimetal phases, respectively. Post treatment surface mineral phase analysis indicates

$23 \mathrm{Mg}(\mathrm{OH})_{2}$ as the main oxidized species; oxide formation does not appear to impair treatment.

24

25 Keywords: bimetal, magnesium, insensitive munition, reduction, DNAN, wastewater

Distribution A: Approved for Public Release; Distribution is Unlimited 


\section{Introduction}

The quest for safe munitions has led to the development of new formulations, designated as insensitive munitions (IMs), based on components that are less prone to accidental detonation. Manufacturing and handling of these IMs generate waste streams containing mixtures of IMs and their manufacturing and transformation byproducts that require further treatment. One specific IM component, 2,4-dinitroanisole (DNAN) has seen heavy use, and thus has garnered research interests in different treatment methods to degrade this target compound in waste streams.

Degradation of pure DNAN by ZVI (Hawari et al., 2015) and its photodegradation (Rao et al., 2013a; Arthur et al., 2017) have been reported. In addition, extensive research on the degradation of DNAN in IM wastewater has been conducted. These studies have evaluated various technologies including: phytoremediation (Shih et al., 2009), aerobic biodegradation (Fida et al., 2014), ZVI/Fenton treatment (Liu et al., 2015), ZVI/anaerobic digestion (Ahn et al., 2011), $\mathrm{Fe} / \mathrm{Cu}$ bimetal/Fenton treatment (Shen et al., 2013), and reduction by $\mathrm{Fe} / \mathrm{Cu}$ (Koutsospyros et al., 2012; Kitcher et al., 2017). Treatment of DNAN by bimetals typically exhibits several advantages when compared to other technologies including extremely rapid degradation kinetics leading to high removal efficiency. DNAN degradation with Fe-based bimetals exhibited fast degradation with complete removal in several minutes (Kitcher et al., 2017). Although treatment of DNAN with Fe-based reagents (ZVI or bimetals) has been demonstrated, the potential use of a similar reagent (i.e. Mg-based bimetals) has not yet been explored.

The bimetal technology is based on enhancing the reactivity of a zero-valent base metal by close contact (i.e. coating) with a catalytic metal to create a galvanic cell. Both $\mathrm{Mg}$ and ZVI have been combined with various catalytic metals to produce reductive bimetal systems that have 
49 treated effectively halogenated compounds and nitro-based explosives (Morales et al., 2002;

50 DeVor et al., 2009; Begum and Gautam, 2011; Koutsospyros et al., 2012; Liu et al., 2015).

51 Specifically, Mg-based bimetals are an emerging technology for the treatment of various organic

52 (Gautam and Suresh, 2007; DeVor et al., 2008; Agarwal, Al-Abed and Dionysiou, 2009; Ghauch

53 and Tuqan, 2009) and inorganic contaminants (Ramavandi et al., 2011). Magnesium has

54 attracted additional interest due to its greater electrode potential than iron. In the hydrogenation

55 of phenol to cyclohexane and cyclohexanone, $\mathrm{Mg} / \mathrm{Pd}$ was found more effective than $\mathrm{Fe} / \mathrm{Pd}$, and

$56 \mathrm{Mg}^{0}$ was more effective than $\mathrm{Fe}^{0}$ (Morales et al., 2002). In addition to the selection of a base

57 metal, the choice of catalytic metal can improve treatment effectiveness by increasing the

58 galvanic potential difference between the pair. Some researchers have utilized noble metals to

59 increase the galvanic potential difference such as Pd, Ag, and $\mathrm{Au}$ (Cwiertny et al., 2006; DeVor

60 et al., 2008; Patel and Suresh, 2008; Coutts et al., 2011; Saitta et al., 2015). Catalytic metal

61 selection criteria may be expanded to include economic (e.g. cost), sustainability (e.g. relative

62 abundance, available deposits) and environmental (e.g. regulatory levels) considerations. In this

63 respect, other more inexpensive and readily available metals, such as $\mathrm{Cu}, \mathrm{Ni}$, and $\mathrm{Zn}$ may be

64 attractive alternatives for use in bimetal formulations.

Similar to many other organic compounds, treatment of DNAN by chemical or biological

66 methods may generate transformation byproducts. Identification of byproducts is critical for

67 unveiling the contaminant degradation pathway and establishing that transformed products are

68 toxicologically and environmentally more benign than the parent contaminant. Treatment

69 methods such as photodegradation (Rao et al., 2013b; Hawari et al., 2015; Taylor et al., 2017)

70 and aerobic biodegradation (Fida et al., 2014; Karthikeyan and Spain, 2016) are typically

71 oxidative. Conversely, typical transformation pathways in treatment with ZVI, ZVMg or Fe- and 
72 Mg-based bimetals indicate reductive chemistry, as observed for example in the reduction of

73 nitrate to nitrite (Ileri, Ayyildiz and Apaydin, 2015; Khalil et al., 2016), $\mathrm{Cr}$ (VI) to $\mathrm{Cr}$ (III)

74 (Rivero-Huguet and Marshall, 2009), and the reductive dechlorination of PCBs (Hadnagy, Rauch

75 and Gardner, 2007; Agarwal et al., 2009; Coutts et al., 2011). Reduction of nitro groups by ZVI

76 or Fe-bimetals in various energetics has been demonstrated in the literature. Examples include

77 treatment of 1,3,5-trinitroperhydro-1,3,5-triazine (RDX) by Fe/Cu (Koutsospyros et al., 2012),

78 dinitrophenol (DNP) and dinitrochlorobenzene (DNCB) by $\mathrm{Fe} / \mathrm{Cu}$ (Liu et al., 2015) and DNAN

79 by ZVI and $\mathrm{Fe} / \mathrm{Cu}$ (Ahn et al., 2011; Hawari et al., 2015; Kitcher et al., 2017).

80 In the present work, reductive degradation of DNAN is reported using Mg-based bimetals

81 containing relatively inexpensive and readily available secondary (i.e. catalytic) metals. Three

82 bimetal formulations are evaluated, namely $\mathrm{Mg} / \mathrm{Cu}, \mathrm{Mg} / \mathrm{Ni}$, and $\mathrm{Mg} / \mathrm{Zn}$ and are compared to

83 degradation with ZVMg. The treatment process is evaluated in laboratory prepared DNAN

84 aqueous solutions and in wastewater. Additionally, SEM imaging, EDS, and XRD analyses are

85 used for characterization of the bimetal reagent surface of unused and used particles (i.e. before

86 and after treatment). Furthermore, identification and quantification of byproducts in the

87 dissolved and particulate phases are performed to facilitate carbon mass balance analysis.

Distribution A: Approved for Public Release; Distribution is Unlimited 


\section{Methods}

89

90

91

92

93

94

\subsection{Chemicals and Materials}

Solid magnesium particles (20-230 mesh, reagent grade, 98\% purity), nickel(II) chloride (98\% purity), zinc chloride (98\% purity) and glacial acetic acid $(99 \%+)$ were purchased from Sigma Aldrich (St. Louis, MO). Copper(II) chloride (99\%), acetonitrile (99.5\%, ACS grade), glass fiber filter paper ( $<1$ micron, $55 \mathrm{~mm})$ and nylon filter paper $(0.45$ micron, $55 \mathrm{~mm})$ were purchased from Fisher Scientific (Waltham, MA). Syringe filters (0.45 micron, nylon) were purchased from Achemtek (Worcester, MA). DNAN solids and DNAN, RDX, and NQ (nitroguanidine) standards dissolved in acetonitrile were obtained from Picatinny Arsenal (Wharton, NJ). DNP standard dissolved in methanol, DAAN (2,4-diaminoanisole) solid standard, and 2-ANAN (2amino-4-nitro-anisole, 98\%) were purchased from Sigma Aldrich (St. Louis, MO) and 4-ANAN (2-nitro-4-amino-anisole, 97\%) was purchased from Fisher Scientific. TOC standards were purchased from Fisher Scientific (Waltham, MA). Chemical oxygen demand (COD) kits (TNT 821) and total nitrogen (TN) kits (TNT 826) were purchased from Hach (Loveland, CO).

Photometric analyses using these test kits were performed on a HACH spectrophotometer DR 6000 (Loveland, CO).

The composition of the IM wastewater, obtained from an industrial munitions facility, is reported in Table 1. In addition to DNAN, RDX, NQ, and DNP were also identified and quantified in the wastewater. Additional wastewater characterization included $\mathrm{pH}$, inorganic nitrogen species $\left(\mathrm{NH}_{3}, \mathrm{NO}_{2}, \mathrm{NO}_{3}\right), \mathrm{TN}, \mathrm{COD}$ and TOC. Information on wastewater composition is provided for completeness of information. The target compound for the present work is only DNAN.

Distribution A: Approved for Public Release; Distribution is Unlimited 
Table 1. DNAN Wastewater Characteristics ${ }^{1}$

111

112

113

114

115

116

117

118

119

120

121

122

123

124

125

126

127

128

129

130

\begin{tabular}{|c|c|c|c|c|c|c|c|c|c|c|}
\hline $\begin{array}{c}\text { RDX } \\
\left(\mathrm{mg} \mathrm{L}^{-1}\right)\end{array}$ & $\begin{array}{c}\mathrm{NQ} \\
\left(\mathrm{mg} \mathrm{L} \mathrm{L}^{-1}\right)\end{array}$ & $\begin{array}{c}\text { DNAN } \\
\left(\mathrm{mg} \mathrm{L}^{-1}\right)\end{array}$ & $\begin{array}{c}\text { DNP } \\
\left(\mathrm{mg} \mathrm{L}^{-1}\right)\end{array}$ & $\mathbf{p H}$ & $\begin{array}{c}\mathrm{NH}_{3}-\mathrm{N} \\
\left(\mathrm{mg} \mathrm{L}^{-1}\right)\end{array}$ & $\begin{array}{c}\mathrm{NO}_{2-\mathrm{N}} \\
\left(\mathrm{mg} \mathrm{L}^{-1}\right)\end{array}$ & $\begin{array}{c}\mathrm{NO}_{3}-\mathrm{N} \\
\left(\mathrm{mg} \mathrm{L}^{-1}\right)\end{array}$ & $\begin{array}{c}\text { TN } \\
\left(\mathrm{mg} \mathrm{L}^{-1}\right)\end{array}$ & $\begin{array}{c}\text { COD } \\
\left(\mathrm{mg} \mathrm{L}^{-1}\right)\end{array}$ & $\begin{array}{c}\text { TOC } \\
\left(\mathrm{mg} \mathrm{L}^{-1}\right)\end{array}$ \\
\hline 5 & 0.5 & 110 & 150 & 7.08 & B.D.L. & B.D.L. & 3.08 & 47 & 470 & 120 \\
\hline
\end{tabular}

${ }^{1}$ B.D.L. $=$ below detection limit

\subsection{Treatment Experiments}

Completely mixed laboratory batch experiments were conducted to evaluate the efficacy

of DNAN treatment using select Mg-based bimetals. All experiments were carried out in $40 \mathrm{~mL}$ VOA vials using a $22 \mathrm{~mL}$ reaction volume at $0.5 \%$ solids/liquid $(\mathrm{S} / \mathrm{L})$ ratio and $10: 1 \mathrm{Mg}$ to secondary metal (i.e. $\mathrm{Cu}, \mathrm{Ni}, \mathrm{Zn}$ ) ratio. Additional experiments for byproducts and mass balance used different reaction volumes $(15-132 \mathrm{~mL})$ with the same $\mathrm{S} / \mathrm{L}$ ratio and all other identical conditions. The $0.5 \% \mathrm{~S} / \mathrm{L}$ ratio was chosen based on previously reported work on DNAN degradation using Fe-based bimetals (Koutsospyros et al., 2012). The 10:1 Mg to secondary metal ratio was decided based on several other studies using $\mathrm{Mg}$ in bimetal formulations. $\mathrm{Mg} / \mathrm{Cu}$ was used in 10:1 ratio to treat azo dye (Asgari, Ramavandi and Farjadfard, 2013), and endosulfan and lindane were treated with $\mathrm{Mg} / \mathrm{Pd}$ at 7.5:1 and 5:1 ratios (Begum and Gautam, 2011) and at 50:1 ratio (Aginhotri, Mahidrakar and Gautam, 2011). In the present study, $0.11 \mathrm{~g}$ of $\mathrm{Mg}$ granules, $10 \mathrm{~mL}$ of water and $1 \mathrm{~mL}$ of catalytic metal solution $(22.27,24.29,22.94 \mathrm{~g} / \mathrm{L}$ for $\mathrm{CuCl}_{2}, \mathrm{NiCl}_{2}$, and $\mathrm{ZnCl}_{2}$, respectively) prepared in deionized water were combined and mixed on a magnetic stirrer plate (Color Squid model, IKA, Wilmington, NC) at a mixing speed of 500 rpm for $5 \mathrm{~min}$. In experiments with $\mathrm{ZVMg}$, the volume of catalytic metal was replaced with additional DI water. The treatment was initiated by adding $10 \mathrm{~mL}$ of DNAN wastewater or a 250 $\mathrm{mg} \mathrm{L}^{-1}$ pure DNAN aqueous solution. After $2.5 \mathrm{~h}$ treatment, an aliquot of the dissolved phase was analyzed by filtering the mixture with a nylon syringe filter ( 0.45 micron, Achemtek).

Distribution A: Approved for Public Release; Distribution is Unlimited 
132 Mass balance experiments were performed to measure dissolved, adsorbed and volatilized TOC 133 and were carried out under identical treatment conditions (aqueous solutions, 0.5\% S/L, $2.5 \mathrm{hr}$ 134 treatment time, and 10:1 Mg to catalytic metal ratio). However, dissolved TOC measurements 135 were performed in experiments scaled by 3 (i.e. $66 \mathrm{~mL}$ total rather than $22 \mathrm{~mL}$ in previous 136 experiments). Adsorbed TOC was measured indirectly by acid digestion of the entire reaction 137 mixture (i.e. treated solution and bimetal together). Therefore, the TOC adsorbed to the bimetal 138 could be determined by subtracting the dissolved TOC from the combined adsorbed and 139 dissolved TOC measurements. Acid digestions were performed by the addition of $1 \mathrm{~mL}$ of 140 sulfuric acid (technical grade, 95\% purity). Additionally, experiments for adsorbed TOC 141 measurements used an adjusted synthesis step that used less water (i.e. $5 \mathrm{~mL}$ instead of the 142 previous $11 \mathrm{~mL}$ ). Volatilized TOC were analyzed qualitatively by GC-MS by capturing the gas 143 in multilayer foil gas bags (Supelco, Bellefonte, PA), however gaseous species were not 144 detected.

\subsection{Analytical Methods}

146 DNAN was analyzed by reversed phase high pressure liquid chromatography (HPLC) on an 147 Agilent 1260 HPLC instrument (Santa Clara, CA) equipped with a Grace Alltech Adsorbosphere 148 HS C-18 $(5 \mu \mathrm{m}, 250 \times 4.6 \mathrm{~mm})$ and a DAD detector (i.e. HPLC-DAD). The mobile phase was an 149 isocratic mixture of methanol:water at 70:30 (v/v), pumped at $1 \mathrm{~mL} \mathrm{~min}^{-1}$; the injection volume 150 was $30 \mu \mathrm{L}$ of sample; the analytical wavelength was $300 \mathrm{~nm}$ (optimal absorbance wavelength for 151 DNAN). At these conditions, DNAN eluted at $4.1 \mathrm{~min}$. Quantification of 2,4-dinitrophenol (DNP) on HPLC was based on an isocratic flow using 153 a solvent of $20 \%$ methanol and $80 \%$ water at a flow rate of $1 \mathrm{~mL} \mathrm{~min}^{-1}$; DNP eluted at $2.5 \mathrm{~min}$. 
154 Simultaneous measurements of DNAN, 2-ANAN, 4-ANAN and DAAN were performed on the 155 same column and detector. A separate analytical method was developed for this analysis, which 156 used a 5 min hold of 90:10 water-methanol mobile phase, followed by a 50 min gradient to $10 \%$ 157 water, $90 \%$ methanol and with a 5 min hold of $10 \%$ water, $90 \%$ methanol pumped at a flow rate 158 of $1 \mathrm{~mL} \mathrm{~min}^{-1}$. At these conditions, the elution times were: DAAN at $5.5 \mathrm{~min}, 4-\mathrm{ANAN}$ at 19 $159 \min , 2-A N A N$ at $26 \mathrm{~min}$, and DNAN at $32 \mathrm{~min}$. The analytical wavelength used was $254 \mathrm{~nm}$ (a 160 wavelength at which all four compounds of interest absorb well). The gradient method was used 161 due to greatly different hydrophobicity and, therefore, significantly different retention times of 162 DAAN and DNAN.

TOC was measured via a UV-Persulfate TOC Analyzer Phoenix 8000 instrument from

164 Teledyne Tekmar (Mason, OH). Identification of byproducts was performed using electrospray 165 ionization tandem mass spectrometry (ESI-MS/MS) on a Waters Quattro Ultima (Milford, MA), 166 i.e. through direct injection of the sample without any separation. Analyses were performed in 167 both positive and negative ionization modes. Tandem mass spectrometry (MS/MS) was essential 168 due to the injection of mixtures (e.g. treated pure compound generating several byproducts) and 169 also in acquiring the necessary daughter spectra for compound identification. ESI-MS, combined 170 with front-end separation with HPLC (i.e. HPLC-ESI-MS), was used for additional confirmation

171 (HPLC: Agilent 1100 Series, Santa Clara, CA; MS: Waters Micromass ZQ instrument, Milford, 172 MA).

\subsection{Surface Characterization}

174 XRD patterns were acquired on a Rigaku Ultima IV X-Ray diffractometer (The Woodlands,

175 TX). Scans acquired were from 5 to $652 \theta$ with an increment of $0.03 \theta$ and scan speed of 2

176 seconds. The x-ray conditions were $40 \mathrm{kV}$ and $40 \mathrm{~mA}$. These were the recommended standard 
177 method and conditions according to the manufacturer. SEM images were obtained with focus ion

178 beam scanning electron microscopy (FIB-SEM), and EDS analyses were performed with a

179 silicon drift detector (SDD) both on a Zeiss Auriga instrument (Oberkochen, Germany).

180 The surface of the bimetals was examined by SEM, XRD, and EDS analyses both before and

181 after treatment, referred to as unused and used particles, respectively. One sample for each

182 bimetal (i.e. $\mathrm{Mg} / \mathrm{Cu}, \mathrm{Mg} / \mathrm{Zn}$, and $\mathrm{Mg} / \mathrm{Ni}$ ) was prepared. Unused particles were synthesized under 183 the same conditions as used ones except without the addition of DNAN. Treated bimetal solids

184 were separated from the liquid by vacuum filtration and allowed to dry on glass slides for 30-60

185 min. Unused samples were decanted and dried overnight to ensure complete dryness.

3. Results and Discussion

\subsection{Bimetal Synthesis and Characterization}

Bimetal particles were synthesized in this work and, therefore, surface characterization was required to ensure that the catalytic metal had coated the base metal. SEM imaging with backscatter detection allowed the detection of the heavier catalytic metals (i.e. $\mathrm{Cu}, \mathrm{Ni}$, and $\mathrm{Zn}$ ), 192 which appeared brighter than the less heavy base metal Mg on the images. For each bimetal configuration, successful coating of the base metal by the catalytic metal was observed. Solid $\mathrm{Cu}$

194 nanoparticles $(<100 \mathrm{~nm})$ coated the $\mathrm{Mg}$ (Figure 1a). A contrasted and zoomed-in image allowed better observation of the bright $\mathrm{Cu}$ nanoparticles (Figure S.1). Other studies that evaluated the

$196 \mathrm{Mg} / \mathrm{Pd}$ bimetal found small islands of Pd deposits (i.e. 50-100 nm) on the $\mathrm{Mg}$ surface using the 197 same imaging technique (Agarwal, Al-Abed and Dionysiou, 2007). In the present work, solid Zn 198 was coated on the Mg in the form of larger micron-sized particles (Figure 1b). This figure is 199 presented at smaller magnification in order to optimally view the $\mathrm{Zn}$ deposits against the $\mathrm{Mg}$ 
200 base metal. In contrast to the $\mathrm{Cu}$ and $\mathrm{Zn}$ particles, $\mathrm{Ni}$ was observed to coat the $\mathrm{Mg}$ uniformly, an

201 observation supported by the lack of distinct structures on the surface of the $\mathrm{Mg} / \mathrm{Ni}$ bimetal

202 (Figure 1c).

203

204 Fig. 1 SEM images of catalytic metal coating on the $\mathrm{Mg}$ base metal: (a) $\mathrm{Mg} / \mathrm{Cu}$ : $\mathrm{Cu}$ nanoparticles 205 ('bright spots'), (b) $\mathrm{Mg} / \mathrm{Zn}$ : micron-sized Zn deposits, and (c) $\mathrm{Mg} / \mathrm{Ni}$ : uniform Ni coating 206

\subsection{Degradation of Pure DNAN in the Aqueous Phase}

The degradation of the target compound DNAN was first examined in laboratory-made aqueous solution. This was done to isolate the behavior of the compound from the wastewater matrix. Treatment of aqueous solutions of pure DNAN with any of the three bimetals resulted in significantly higher extent of removal compared to that of ZVMg alone, i.e. without the addition

212 of a catalytic metal (Figure 2). The treatment efficiency of the $\mathrm{Mg} / \mathrm{Cu}, \mathrm{Mg} / \mathrm{Zn}$ and $\mathrm{Mg} / \mathrm{Ni}$

213 bimetal configurations was $100 \%, 95 \%$ and $89 \%$ removal, respectively. ZVMg performed poorly

214 at a removal efficiency of 35\%. Poor removal efficiencies of ZVMg and ZVI with systems near

215 neutral $\mathrm{pH}$ have been reported for nitrate and $\mathrm{Cr}(\mathrm{VI})$ reduction, by Khalil et al. (2016) and

216 Rivero-Huguet et al. (2009). Furthermore, enhancement of reductive degradation by addition of a 217 catalytic salt has been reported for Fe-based bimetals (Rivero-Huguet and Marshall, 2009; Xiong 218 et al., 2015; Khalil et al., 2016) and Mg-based bimetals (Solanki and Murthy, 2011; Saitta et al., 2192015 ) for various inorganic and organic contaminants. In the present work, degradation by 220 ZVMg was evidently similarly enhanced with the addition of the catalytic metal.

222 Fig. 2 Pure DNAN removal (\%) in the aqueous phase after bimetal treatment (0.5\% S/L, 10:1

$223 \mathrm{Mg}$ to catalytic metal ratio, and $2.5 \mathrm{~h}$ treatment time) compared to $\mathrm{ZVMg}$ treatment Distribution A: Approved for Public Release; Distribution is Unlimited 
After treatment with ZVMg and the bimetals, the final $\mathrm{pH}$ was higher than that of the

original DNAN aqueous solution. This was likely due to the consumption of protons $\left(\mathrm{H}^{+}\right)$during

227 the chemical reduction (Begum and Gautam, 2011; Khalil et al., 2016). Furthermore, treatment

228

with any of the three bimetals equilibrated to a final $\mathrm{pH}$ in the range of 9.9-10.2, while treatment

229

with ZVMg resulted in a higher final $\mathrm{pH}$ of 10.7 (Table 2). Reduction of nitrate by ZVI also

230

generated a higher $\mathrm{pH}$ than treatment by $\mathrm{Fe} / \mathrm{Cu}$ (Khalil et al., 2016). Oxidized species of $\mathrm{Cu}$ and

231

Fe (i.e. $\mathrm{CuFe}_{2} \mathrm{O}_{4} * \mathrm{Fe}_{3} \mathrm{O}_{4}$ ) had formed, and these side reactions likely generated protons; therefore,

232

the final $\mathrm{pH}$ was lower in the $\mathrm{Fe} / \mathrm{Cu}$ treated system. Similar reactions may have occurred during

233

reduction with $\mathrm{ZVMg}$ versus a $\mathrm{Mg}$-bimetal in the present study, i.e. side reactions during the

234 formation of oxidized metal species of the base metal $\mathrm{Mg}$ and/or catalytic metal may generate $\mathrm{H}^{+}$

235 thereby reducing the $\mathrm{pH}$.

Table 2. Parameters of DNAN Treatment in Two Matrices*

\begin{tabular}{|c|c|c|c|c|c|c|}
\hline \multirow{3}{*}{ Matrix } & System & Initial pH** & Final pH & $\begin{array}{c}\text { Initial } \\
\text { ORP** } \\
(\mathbf{m V})\end{array}$ & $\begin{array}{c}\text { Final } \\
\text { ORP } \\
(\mathbf{m V})\end{array}$ & $\begin{array}{c}\text { DNAN } \\
\text { Removal } \\
(\%)\end{array}$ \\
\hline \multirow{6}{*}{} & Control & $5.70( \pm 0.10)$ & $5.01( \pm 0.34)$ & $76( \pm 44)$ & $171( \pm 34)$ & $1.6( \pm 0.5)$ \\
\cline { 2 - 7 } & $\mathrm{ZVMg}$ & $9.77( \pm 0.64)$ & $10.72( \pm 0.10)$ & $82( \pm 36)$ & $24( \pm 8)$ & $35.1( \pm 4.0)$ \\
\cline { 2 - 7 } & $\mathrm{Mg} / \mathrm{Cu}$ & $9.42( \pm 0.53)$ & $10.20( \pm 0.06)$ & $-96( \pm 20)$ & $-96( \pm 12)$ & $100.0( \pm 0.0)$ \\
\cline { 2 - 7 } & $\mathrm{Mg} / \mathrm{Ni}$ & $7.92( \pm 0.08)$ & $9.91( \pm 0.08)$ & $-16( \pm 27)$ & $-108( \pm 22)$ & $88.8( \pm 3.6)$ \\
\cline { 2 - 7 } & $\mathrm{Mg} / \mathrm{Zn}$ & $7.03( \pm 0.36)$ & $10.22( \pm 0.05)$ & $27( \pm 11)$ & $-7( \pm 7)$ & $94.7( \pm 1.6)$ \\
\hline \multirow{6}{*}{$\mathrm{Control}$} & $6.64( \pm 0.01)$ & $7.15( \pm 0.09)$ & $196( \pm 64)$ & $199( \pm 10)$ & $6.2( \pm 0.3)$ \\
\cline { 2 - 7 } & $\mathrm{ZVMg}$ & $10.18( \pm 0.06)$ & $10.86( \pm 0.06)$ & $-131( \pm 20)$ & $36( \pm 4)$ & $12.9( \pm 2.0)$ \\
\cline { 2 - 7 } & $\mathrm{Mg} / \mathrm{Cu}$ & $10.43( \pm 0.07)$ & $9.99( \pm 0.08)$ & $-295( \pm 37)$ & $-225( \pm 8)$ & $100.0( \pm 0.0)$ \\
\cline { 2 - 7 } & $\mathrm{Mg} / \mathrm{Ni}$ & $7.96( \pm 0.07)$ & $9.93( \pm 0.09)$ & $-231( \pm 27)$ & $-188( \pm 20)$ & $97.2( \pm 0.4)$ \\
\cline { 2 - 7 } & $\mathrm{Mg} / \mathrm{Zn}$ & $8.32( \pm 0.57)$ & $10.15( \pm 0.10)$ & $-108( \pm 55)$ & $-158( \pm 18)$ & $90.5( \pm 3.5)$ \\
\hline
\end{tabular}

*Treatment time of $2.5 \mathrm{~h}$

**Initial $\mathrm{pH}$ and initial ORP obtained for treated samples were measurements taken immediately after contact between DNAN and the reagents had been established 
242

243

244

245

246

247

248

249

250

251

252

253

254

255

256

257

258

259

260

261

262

263

264

265

\subsection{Reaction Byproduct Identification in the Dissolved Phase}

To shed light to the Mg-based reductive degradation of DNAN, it is critical to identify

and quantify the reaction products formed. Mass spectra were acquired from treated samples in both the aqueous solution and wastewater experiments using ESI-MS/MS and HPLC-ESI-MS in positive and negative ionization modes. Detection of products at the attempted initial DNAN concentration was not possible due to low concentrations close to detection levels. Since higher initial DNAN concentrations could not be pursued due to aqueous solubility limitations, experiments were set up using an alternative solvent. Products were, however, identified under different conditions: 1) treatment of pure DNAN in an acetonitrile solvent matrix and 2) treatment of an identified byproduct, 4-ANAN, in DI water. Products were characterized only for $\mathrm{Mg} / \mathrm{Cu}$ treatment.

\subsubsection{DNAN Treated in a Solvent Matrix}

A solvent matrix (i.e. ACN) was used to produce higher initial DNAN concentrations, which led to higher, detectable concentrations of byproducts. Treatment conditions were identical to those of previously mentioned experiments (i.e. $0.5 \% \mathrm{~S} / \mathrm{L}$ ratio, $10: 1 \mathrm{Mg}$ to $\mathrm{Cu}$ ratio) except that the initial stock solution contained $1,350 \mathrm{mg} \mathrm{L}^{-1} \mathrm{DNAN}$ in $\mathrm{ACN}$ as opposed to the earlier $250 \mathrm{mg} \mathrm{L}^{-1}$ DNAN in water. In ACN, the daughter spectrum of m/z 169 in positive ionization mode produced fragmentation that indicated the production of 2-ANAN and 4-ANAN during treatment (Figure 3a). The daughter spectrum of $\mathrm{m} / \mathrm{z} 139$ produced fragmentation that indicated the formation of DAAN (Figure S.2a). Reference spectra were acquired from aqueous solutions of 2ANAN, 4-ANAN (Figure 3b, c) and DAAN (Figure S.2b) to confirm their presence in the treated DNAN sample. The peaks at m/z 169 for the 2-ANAN and 4-ANAN spectra and at m/z 139 for the DAAN spectrum were identified as the protonated species, i.e. $[\mathrm{M}+\mathrm{H}]^{+}$, because the nominal masses of the neutral molecules are 168 and $138 \mathrm{Da}$, respectively. In negative ionization mode, 
266 no other significant peaks, other than those corresponding to 2-ANAN, 4-ANAN or DAAN, 267 were observed.

268 Fig. 3 Daughter spectrum of m/z 169 from ESI-MS/MS in positive mode from (a) after DNAN 269 treatment (solvent matrix, 0.5\% S/L, 10:1 Mg to Cu ratio, 2.5 hour treatment time), (b) pure 2270 ANAN reference, and (c) pure 4-ANAN reference. The difference in maximum intensities are

271 neglected as comparison of relative intensities were required

272

273 DAAN was also detected when DNAN was reduced with ZVI by Hawari et al. (2015) and Ahn 274 et al. (2011) and with Fe/Cu by Liu et al., 2015 (Ahn et al., 2011; Hawari et al., 2015; Liu et al., 275 2015). Ahn et al. (2011) also identified both 2-ANAN and 4-ANAN similarly to the present 276 work (Ahn et al., 2011), while Hawari et al. (2015) only detected 2-ANAN as byproducts of 277 DNAN degradation. Hawari et al. (2015) attributed the fact that only 2-ANAN was detected in 278 their study to the regioselectivity of reduction in the ortho position, i.e. reduction more favorably 279 produced 2-ANAN over 4-ANAN; therefore, 4-ANAN generated was below the detection limit 280 (Hawari et al., 2015). Kitcher et al. (2017) confirmed reduction of nitro groups to amino groups 281 by in source deuterium exchange, which indicated m/z 169 as an amino product; it was assumed 282 that $\mathrm{m} / \mathrm{z} 169$ meant both ortho and para reduction occurred, i.e. both 2-ANAN and 4-ANAN 283 were formed (Kitcher et al., 2017). In the present study, the analysis of daughter spectra revealed that 2-ANAN produced 285 mass spectral peaks of m/z (in positive mode): 169, 154, and 123. 4-ANAN produced mass 286 spectral peaks of $\mathrm{m} / \mathrm{z}: 169,154,152,123,122$, and 94 . The low intensity of $\mathrm{m} / \mathrm{z} 152$ in the 287 treated sample (Figure 3a) indicates that a mixture of both 4-ANAN and 2-ANAN was present. 288 For example, if only 4-ANAN were present, the intensity of m/z 152 peak would be higher, 
289

290

291

292

293

294

295

296

297

298

299

300

301

302

303

304

305

306

307

308

309

310

311

while if only 2-ANAN were present, there would be no peak at $\mathrm{m} / \mathrm{z} 152$. However, there is a minute peak of $\mathrm{m} / \mathrm{z} 152$ in the mass spectrum (Figure 3a). In addition, the same was observed with the relative intensity of the $\mathrm{m} / \mathrm{z} 123$ and 122 peaks. If only 4-ANAN were present, the $\mathrm{m} / \mathrm{z}$ 122 peak in Figure 3a would be relatively higher to that of $\mathrm{m} / \mathrm{z}$ 123, i.e. the two peaks should match the relative heights of the m/z 122 and 123 peaks in the 4-ANAN reference. Ultimately, the presence of 2-ANAN effectively suppressed the intensity of the m/z 122 peak when mixed with 4-ANAN. Olivares et al. (2016) was also able to differentiate between the 2-ANAN and 4ANAN isomers based on their unique fragmentation patterns. Furthermore, Hawari et al. (2015) detected a hydroxylamino intermediate (i.e. 2-HA-NAN) and small amounts of a nitroso intermediate (i.e. 2-NO-NAN). According to Hudlicky (1984), reduction of the nitro group in nitroaromatic compounds follows the scheme: nitro $\left(-\mathrm{NO}_{2}\right)>$ nitroso $(-\mathrm{NO})>$ hydroxylamino $(-$ $\mathrm{NHOH})>$ amine $\left(-\mathrm{NH}_{2}\right)$, according to the degree of reduction. Nitroso and hydroxylamino compounds are typically rarely observed under this scheme (Hudlicky, 1984). In this study, no nitroso or hydroxylamino intermediates in either the ortho or para positions were identified.

\subsubsection{Reduction of 4-ANAN to DAAN}

The high aqueous solubility of 4-ANAN $\left(4,400 \mathrm{mg} \mathrm{L}^{-1}\right.$ at $25^{\circ} \mathrm{C}$ (Hawari et al., 2015)), an identified byproduct of DNAN degradation in the ACN matrix, allowed investigation of further byproduct formation directly in aqueous solutions, i.e. as opposed to work performed in an ACN matrix. The reduction of 4-ANAN to DAAN was confirmed; treatment of 4-ANAN (800 mg L-1 initial concentration, $0.5 \% \mathrm{~S} / \mathrm{L}, 10: 1 \mathrm{Mg}$ to $\mathrm{Cu}$ ratio, 1 hour treatment time) yielded DAAN (m/z 139) (Figure 4). The presence of DAAN was additionally confirmed on HPLC-ESI-MS for the same treatment condition (Figure S.3). Adding front-end separation with HPLC to the MS method allowed the matching of elution times of pure DAAN (observed as $\mathrm{m} / \mathrm{z} 139$ ) to that of 
312 the m/z 139 peak in treated 4-ANAN. Although the reduction of 4-ANAN to DAAN has been

313 proposed as part of the DNAN degradation pathway using ZVI (Ahn et al., 2011; Hawari et al.,

$3142015)$ and $\mathrm{Fe} / \mathrm{Cu}$ (Kitcher et al., 2017), the same has not yet been reported for Mg-based bimetal

315 treatment in the literature. Additionally, the detection of DNAN reduction to DAAN by any

316 bimetal has not yet reported until now; Kitcher et al. (2017) proposed DAAN as a probable final

317 product by Fe-bimetal reduction, but could not confirm analytically.

Fig. 4 Daughter spectrum of m/z 139 from ESI-MS/MS in positive mode of 4-ANAN treated 320 with $\mathrm{Mg} / \mathrm{Cu}$ (DI water matrix, $0.5 \% \mathrm{~S} / \mathrm{L}, 10: 1 \mathrm{Mg}$ to $\mathrm{Cu}$ ratio, $1 \mathrm{hr}$ treatment time) showing 321 DAAN (m/z 139) with matching fragmentation of pure DAAN (Figure S.2b)

322

323

324

325

326

327

328

329

330

331

332

333

334

\subsection{Reaction Pathway}

The proposed partial reaction pathway for DNAN reduction by $\mathrm{Mg} / \mathrm{Cu}$ is: 1) reduction of one nitro group, either ortho or meta position, which forms 2-ANAN or 4-ANAN, and then 2) subsequent reduction of the other nitro group, which forms DAAN (Figure 5). This pathway was identical to the one identified for DNAN reduction with ZVI (Ahn et al., 2011) and $\mathrm{Fe} / \mathrm{Cu}$ (Kitcher et al., 2017). In the present study, the byproducts and reaction pathways characterized to-date were from DNAN treatment with $\mathrm{Mg} / \mathrm{Cu}$, and it is likely that identical pathways exist for treatment with $\mathrm{Mg} / \mathrm{Ni}$ and $\mathrm{Mg} / \mathrm{Zn}$. The proposed pathway excludes the unstable nitroso and hydroxylamine derivatives discussed earlier since these compounds were not detected.

Fig. 5 Partial reaction pathways of DNAN degradation by $\mathrm{Mg} / \mathrm{Cu}$ 
335 The reduction of DNAN to byproducts 2-ANAN, 4-ANAN and DAAN were made possible by

336 electrons released by the dissolution of $\mathrm{Mg}^{0}$ as illustrated in Figure 6. In the bimetal pair, $\mathrm{Mg} / \mathrm{Cu}$,

$337 \mathrm{Cu}$ is the cathode while $\mathrm{Mg}$ is the anode. $\mathrm{Mg}$ thus preferentially corrodes to $\mathrm{Mg}^{2+}$ with the

338 concurrent release of 2 electrons. Electrons become available for reduction illustrated by the

339 dashed lines. In addition, $\mathrm{Mg}(\mathrm{OH})_{2}$ forms by the oxidation of $\mathrm{Mg}$ in water (Figure 6). The same

340 reactions occur in the case of $\mathrm{Mg} / \mathrm{Ni}$ and $\mathrm{Mg} / \mathrm{Zn}$, where $\mathrm{Ni}$ and $\mathrm{Zn}$ are cathodic relative to the $341 \mathrm{Mg}$.

342

343 Fig. 6 Diagram illustrating the galvanic corrosion of $\mathrm{Mg}$ in the $\mathrm{Mg} / \mathrm{Cu}$ bimetal pair resulting in 344 reduction of DNAN and oxidation of $\mathrm{Mg}$ to $\mathrm{Mg}(\mathrm{OH})_{2}$

345

346

347

348

349

350

351

352

353

354

355

356

\subsection{Quantification of Reaction Byproducts and Mass Balance}

Mass balance experiments are necessary in order to fully characterize any treatment system. A significantly open mass balance means undetected compounds or unknown fates, with potential to toxic exposure. In the present study, preliminary mass balance data were obtained for treatment of pure DNAN in water and in $\mathrm{ACN}$ with $\mathrm{Mg} / \mathrm{Cu}$. Since byproducts of pure DNAN were not detected in the aqueous (i.e. dissolved) phase, the TOC mass balance was evaluated (see Section 3.5.1). Next, experiments with pure DNAN were performed in ACN and the mass balance of DNAN and its known reaction products was evaluated in the combined dissolved and adsorbed phase (obtained by acid digestion of the reaction mixture), with subsequent quantification of individual known compounds in the dissolved phase (see Section 3.5.2).

3.5.1 Overall Carbon Mass Balance Closure Using Aggregate TOC Measurements

Distribution A: Approved for Public Release; Distribution is Unlimited 
357 After treatment of pure DNAN with $\mathrm{Mg} / \mathrm{Cu}$, a $91 \%$ carbon mass balance closure was attained by

358 addition of the dissolved and adsorbed phases (i.e. adsorbed to the bimetal surface). The

359 dissolved phase and adsorbed phase contained $12.4 \%$ and $78.4 \%$ of TOC, respectively, compared

360 to the control. On the other hand, treatment of DNAN by ZVI resulted in $95 \%$ of the initial

361 DNAN mass recovered as DAAN in the aqueous phase after 1 hour of treatment (Ahn et al.,

362 2011). This observed difference of mass balance between ZVI in the study of Ahn et al. (2011)

363 and $\mathrm{Mg} / \mathrm{Cu}$ in the present work might stem from increased oxidization of the $\mathrm{Mg}$-bimetal from

364 galvanic corrosion. The enhanced corrosion of the Mg surface during treatment may have

365 increased the total surface area and therefore increased the number of sites available for 366 adsorption.

367 This significant adsorption was validated by analysis of the sealed reactor headspace with 368 GC-MS in experiments conducted with aqueous solutions of pure DNAN. A significant amount 369 of gas (approximately $0.5-1.0 \mathrm{~L}$ ) was generated (with reaction volumes of $132 \mathrm{~mL}$ ), but no 370 reaction byproducts were identified. The gas captured was speculated to be mainly $\mathrm{H}_{2}$ gas, an 371 expected gas formed in the dissolution of $\mathrm{Mg}$ or Fe in water (Patel and Suresh, 2007; Lee and 372 Park, 2013; Nie et al., 2013). 3.5.2 Mass Balance of Dissolved Byproducts: Treatment in Solvent Matrix

374 Byproducts in the dissolved phase have been qualitatively detected. However, quantification of 375 dissolved byproducts was necessary to close the mass balance in the liquid phase to ensure that 376 the products detected (2-ANAN, 4-ANAN and DAAN) accounted for all dissolved TOC.

377 Significant open mass balance in the liquid phase would indicate formation of additional 378 unidentified products. Byproducts in the dissolved phase after DNAN treatment in aqueous 379 solutions could not be detected by MS or HPLC. However, byproducts 2-ANAN and 4-ANAN 
380 were detected in small amounts for DNAN treatment by $\mathrm{Mg} / \mathrm{Cu}$ in $\mathrm{ACN}$ (Figure 7). Furthermore, 381 dissolved DAAN was detected on ESI-MS, but was not detected on the HPLC-DAD used for 382 quantification, and therefore the bar for DAAN was marked with an asterisk $(*)$ to indicate future 383 work is needed for analytical method development for this compound. Mass balance data from 384 aggregate measurements after treatment in the solvent matrix could not be supplemented with 385 COD and TOC removals due to the significant addition of ACN. Ultimately, the small amounts 386 of 2-ANAN and 4-ANAN found after treatment in the ACN matrix and the undetectable amounts 387 of the same compounds in the aqueous matrix could indicate that DAAN may further degrade to 388 other compounds. Furthermore, the higher concentration of 2-ANAN than that of 4-ANAN on 389 Figure 7 maybe suggestive of regioselectivity of the reduction reaction for the ortho rather than 390 the para isomer. This remains to be resolved and quantified in future work.

392 Fig. 7 Recovered mass of DNAN and products in the dissolved phase after treatment of DNAN 393 with $\mathrm{Mg} / \mathrm{Cu}$ (ACN matrix, 0.5\% S/L, 10:1 $\mathrm{Mg}$ to $\mathrm{Cu}$ ratio, $2.5 \mathrm{hr}$ treatment). Zero amounts of 394 DAAN marked with $(*)$ indicate quantification of this compound requires additional work. 395 Corresponding chromatogram of treated DNAN in upper right; DAAN was not detected on 396 HPLC-DAD

Distribution A: Approved for Public Release; Distribution is Unlimited 


\subsection{Characterization of DNAN Treatment in Wastewater}

Parameters examined for DNAN-laden wastewater treatment included DNAN removal, $\mathrm{pH}$, ORP, TOC and COD reduction, and removal of dinitrophenol (DNP) (DNP was only evaluated qualitatively), another major wastewater constituent. Observed DNAN removals in wastewater were similar to those of in the pure DNAN solutions. In the wastewater, high removals were achieved by $\mathrm{Mg} / \mathrm{Cu}, \mathrm{Mg} / \mathrm{Ni}$, and $\mathrm{Mg} / \mathrm{Zn}$ (i.e. $100 \%, 97 \%$ and $91 \%$, respectively), while treatment with ZVMg resulted in poor degradation efficiency of $12.9 \%$ (Figure 8). However, treatment with $\mathrm{Mg} / \mathrm{Zn}$ resulted in lower DNAN removal than treatment with $\mathrm{Mg} / \mathrm{Ni}$ in wastewater, whereas the opposite trend was observed in aqueous solutions (Table 2).

Fig. 8 DNAN removal (\%) in wastewater after bimetal treatment $(0.5 \% \mathrm{~S} / \mathrm{L}, 10: 1 \mathrm{Mg}$ to catalytic metal ratio, and $2.5 \mathrm{~h}$ treatment time) compared to $\mathrm{ZVMg}$

Based on removal efficiency, the reductive activity of the various bimetal systems was ranked in the following order (treatment of aqueous solutions of pure DNAN):

$\mathrm{Mg} / \mathrm{Cu}>\mathrm{Mg} / \mathrm{Zn}>\mathrm{Mg} / \mathrm{Ni}>\mathrm{ZVMg}$ (Table 2 ). There was no correlation between the final ORP values and the DNAN removal efficiencies. On the other hand, a correlation has been observed between these variables for wastewater experiments. In the wastewater matrix, the final ORP values showed that the $\mathrm{Mg} / \mathrm{Cu}$ bimetal pair exhibited the most negative value $(-225 \mathrm{mV})$, indicating that this system generated the most reductive environment. Based on ORP values, the bimetal systems were ranked in the following order (most negative shown first): $\mathrm{Mg} / \mathrm{Cu}<\mathrm{Mg} / \mathrm{Ni}<\mathrm{Mg} / \mathrm{Zn}<\mathrm{ZVMg}$, which correlated with the DNAN removal efficiency (i.e. higher DNAN removal was achieved under more reducing conditions). The reduction of nitrate by ZVI 
420 and $\mathrm{Fe} / \mathrm{Cu}$ also created a reductive environment as indicated by negative ORP values of around -

$421700 \mathrm{mV}$ (Khalil et al., 2016); the difference in ORP values between reduction with ZVI and

$422 \mathrm{Fe} / \mathrm{Cu}$ was not significantly different. Final $\mathrm{pH}$ values in wastewater were similar to those

423 observed in pure DNAN aqueous solutions: $\mathrm{pH}$ of 9.9-10.2 for the bimetals and somewhat higher

$424 \mathrm{pH}$ of 10.9 for ZVMg (Table 2).

425

426

\subsection{Removal of TOC and COD}

427

The effectiveness of wastewater treatment using bimetals was also measured by removal

428

of aggregate parameters TOC and COD. Aggregate parameters were selected because although

429 the major constituents were DNAN, DNP, and RDX, small amounts of other unknown

430 compounds accounted for approximately $10 \%$ of wastewater TOC.

All three bimetal systems were capable of high DNAN removal of 90-100\% (Figure 8)

432 and TOC and COD removal of $60-70 \%$ (Figure S.4). Empirical measurements of TOC and COD

433 from solutions of DNAN at various concentrations allowed calculation of DNAN-derived TOC

434 and COD. The majority of the remaining TOC and COD after treatment were, therefore, DNAN

435 byproducts and/or byproducts derived from DNP and RDX. In addition, the overall amount of

436 removed TOC and COD exceeded that originating from DNAN. Therefore, the system treated

437 the other organic contaminants as well as DNAN. The remaining TOC and COD may also

438 include species that were more resistant to the bimetal treatment. This was corroborated by a

439 similar level of TOC and COD removal regardless of bimetal configuration and despite the larger

440 DNAN removal capability of $\mathrm{Mg} / \mathrm{Cu}$. $\mathrm{COD}$ removal was not measured for treatment with

441 ZVMg.

442

Furthermore, the degradation of DNP by $\mathrm{Mg} / \mathrm{Cu}, \mathrm{Mg} / \mathrm{Ni}$ and $\mathrm{Mg} / \mathrm{Zn}$, was qualitatively

443 observed by superimposing HPLC chromatograms of wastewater at before and after treatment

Distribution A: Approved for Public Release; Distribution is Unlimited 
$444 \quad(0.5 \% \mathrm{~S} / \mathrm{L}, 10: 1 \mathrm{Mg}$ to catalytic metal ratio) (Figure S.5). The intensity of the chromatographic 445 peak that correlated to DNP (2.1 min) decreased drastically indicating almost complete removal 446 after treatment with each bimetal. The same result was observed for DNAN (4.2 min).

447

448

450

451

452

453

454

455

456

457

458

459

460

461

462

463

464

465

466

\subsection{Effect of Treatment on the Bimetal Surface}

The characterization of the reagent bimetal surface after use was performed to assess any changes to the reagent. For bimetal systems and especially Fe-based bimetals, oxidation of the bimetal surface has led to passivation and inhibition of treatment (Rivero-Huguet and Marshall, 2009; Fu, Cheng and Lu, 2015; Sun et al., 2016). In this work, surface analyses of used Mgbimetal particles included SEM imaging, elemental mapping through EDS, and XRD analysis. EDS results indicated significant elemental oxygen on the surface of all tested bimetal configurations. Therefore, the identification and relative quantification of the mineral species containing oxygen was performed by XRD analysis.

\subsubsection{Elemental Analyses of Oxidation with EDS}

The EDS map of an unused particle of $\mathrm{Mg} / \mathrm{Cu}$ (Figure 9) showed that oxygen was distributed according to the topography of the $\mathrm{Mg}$ surface, which suggested oxidation of the $\mathrm{Mg}$ surface (Figure 9b, c). Therefore, the surface of $\mathrm{Mg} / \mathrm{Cu}$ particles had become oxidized during the bimetal synthesis step before treatment. Oxidation of the surface was likely due to galvanic corrosion after deposition of $\mathrm{Cu}^{0}$. The $\mathrm{Cu}$ nanoparticles were not clearly detectable on EDS mapping (Figure 9d) but were observed on SEM imaging (Figure 1a).

Fig. 9 EDS mapping of (a) a sample region of an unused $\mathrm{Mg} / \mathrm{Cu}$ particle pictured by SEM of an elucidating distribution of (b) primary metal $\mathrm{Mg}$ to (c) oxygen, (d) and catalytic metal $\mathrm{Cu}$

Distribution A: Approved for Public Release; Distribution is Unlimited 
468 After treatment (i.e. on used particles), identification of $\mathrm{Cu}$ nanoparticles on SEM images and

469 EDS mapping was not as certain due to the 'rougher' particle appearance resulting from

470 corrosion (Figure 10a). However, EDS elemental analysis still showed the presence of $\mathrm{Cu}$ on the

471 treated particle (Figure 10d, Table S.1). Additionally, oxidation of the bimetal surface had

472 increased slightly after treatment according to EDS compositional relative quantification (Table

473 S.1). While EDS compositional analyses are generally used for smoother and flat sample

474 surfaces (whereas the analyzed bimetal surfaces have varying topography), the compositions

475 obtained by EDS were still considered one effective measure of overall oxidation as seen in other

476 bimetal literature (Shih et al., 2009; Xu et al., 2012; Nie et al., 2013). In the present study,

477 similar results to that of EDS analyses of $\mathrm{Mg} / \mathrm{Cu}$ particles were found for $\mathrm{Mg} / \mathrm{Ni}$ and $\mathrm{Mg} / \mathrm{Zn}$

478 particles; i.e. oxidation was evident to some extent on the surface of unused and was more

479 pronounced on used particles (Table S.1; EDS elemental mappings of these particles are

480 provided in the supplemental data, Figures S.6-S.9).

481

482 Fig. 10 EDS mapping of (a) SEM of sample region of a used $\mathrm{Mg} / \mathrm{Cu}$ particle elucidating

483 distribution of (b) primary metal $\mathrm{Mg}$ to (c) oxygen, and (d) catalytic metal $\mathrm{Cu}$

484

485

\subsubsection{Identification and Relative Quantification of Oxidized Species}

486 The major oxidized species was identified as $\mathrm{Mg}(\mathrm{OH})_{2}$ through $\mathrm{XRD}$ analysis for all three

487 bimetal configurations. Furthermore, $\mathrm{MgO}$ formation was not observed. The oxidation of the

488 base metal Mg was consistent with galvanic corrosion when in contact with the catalytic metal

489 (i.e. $\mathrm{Cu}, \mathrm{Ni}$ or $\mathrm{Zn}$ ).

Distribution A: Approved for Public Release; Distribution is Unlimited 
The presence of $\mathrm{Mg}(\mathrm{OH})_{2}$ was corroborated on the surface of all three unused bimetal

491 particles, however, to a lesser extent on $\mathrm{Mg} / \mathrm{Ni}$ and $\mathrm{Mg} / \mathrm{Zn}$ than on $\mathrm{Mg} / \mathrm{Cu}$. The reduced

492 formation of $\mathrm{Mg}(\mathrm{OH})_{2}$ on $\mathrm{Mg} / \mathrm{Ni}$ and $\mathrm{Mg} / \mathrm{Zn}$ is in agreement with the lower galvanic potential

493 difference for these bimetal systems compared to $\mathrm{Mg} / \mathrm{Cu}$ (Figure S.10a,b-1 1a,b). After treatment

494 of wastewater, the amount of $\mathrm{Mg}(\mathrm{OH})_{2}$ on the surface of $\mathrm{Mg} / \mathrm{Cu}$ increased significantly (Figure

$49510 \mathrm{a}, \mathrm{b})$, while the opposite trend was observed on the surface of used $\mathrm{Mg} / \mathrm{Ni}$ and $\mathrm{Mg} / \mathrm{Zn}$ particles.

496 This may be due to the unknown ions in the wastewater, which may have solubilized part of the

$497 \mathrm{Mg}(\mathrm{OH})_{2}$ formed on the latter two bimetals. However, in the case of $\mathrm{Mg} / \mathrm{Cu}$, the formation of

498 hydroxide from high galvanic potential difference likely exceeded the rate of $\mathrm{Mg}(\mathrm{OH})_{2}$

499 dissolution due to unknown ions, therefore resulting in the expected increase of hydroxide

500 formation. This is also supported by observations made on the bimetal surfaces after treatment of

501 aqueous solutions of pure DNAN (i.e. a matrix lacking additional ions), where the trend in

502 oxidation of $\mathrm{Mg}$ for each bimetal configuration showed the expected outcome, i.e. the amount of

$503 \mathrm{Mg}(\mathrm{OH})_{2}$ increased after 2.5 hours of treatment (Figures 11c, S.10c, S.11c). Further analysis on

504 the ionic character and composition of the wastewater is required.

505

506 Fig. 11 XRD patterns of $\mathrm{Mg} / \mathrm{Cu}$ (a) before treatment, (b) after treatment in wastewater, and (c)

507 after treatment in aqueous solutions

508

The decrease of $\mathrm{Mg}(\mathrm{OH})_{2}$ on used particles of $\mathrm{Mg} / \mathrm{Ni}$ and $\mathrm{Mg} / \mathrm{Zn}$ may contradict the

510 findings from EDS, which showed that overall oxygen content/oxidation increased. However, it

511 is possible that XRD could not detect other oxidized species since XRD is a technique that

512 detects crystalline materials.

Distribution A: Approved for Public Release; Distribution is Unlimited 
In addition, there were unidentified species (small peaks at 33 and $432 \Theta$ ) that were not

514

515

516

517

518

519

520

521

522

523

524

525

526

527

528

529

530

531

532

533

534

535

536

matched to any oxidized forms of $\mathrm{Cu}$ or $\mathrm{Mg}$ with $\mathrm{Cu}$. In the treatment of nitrate by $\mathrm{Fe} / \mathrm{Cu}(\mathrm{Khalil}$ et al., 2016), species of $\mathrm{Fe}$ and $\mathrm{Cu}$ oxides were detected, specifically $\mathrm{Fe}_{3} \mathrm{O}_{4}$ and $\mathrm{CuFe}_{2} \mathrm{O}_{4}{ }^{\bullet} \mathrm{Fe}_{3} \mathrm{O}_{4}$. However, no other oxidized species of $\mathrm{Cu}$ were detected in the present work. Furthermore, micron-sized deposits of $\mathrm{Zn}^{0}$ were observed, likely due to their larger particle size and increased detection on $\mathrm{XRD} . \mathrm{Zn}^{0}$ was the only zero-valent catalytic metal detected in the present work.

Despite significant oxidation of the $\mathrm{Mg}$ surface, treatment with the Mg-bimetals produced high removals of DNAN. During treatment with ZVI or Fe-bimetals, the formation of (oxy)hydroxides or oxides of iron passivate the surface causing inhibition of treatment $(\mathrm{Fu}$, Cheng and Lu, 2015; Sun et al., 2016). In the treatment with various Fe-bimetal configurations, the passivated Fe-bimetals led to separate modelling of a slower kinetics and "inhibited" reaction rate after an initial period of faster kinetics (Rivero-Huguet and Marshall, 2009). Generally, a low $\mathrm{pH}$ condition for various Fe-based bimetals have been required in order to overcome passivation by oxidation (Xu et al., 2005; Rivero-Huguet and Marshall, 2009; Luo et al., 2010). However, in the present work, an unadjusted $\mathrm{pH}$ condition (Table 2) in each bimetal treatment system still produced effective DNAN removal.

\section{Conclusions}

The preliminary work performed in this study indicates that Mg-based bimetals are effective reagents for the degradation of DNAN and other energetic compounds often present in munitions facilities waste streams. The use of inexpensive catalytic metals to generate effective $\mathrm{Mg}$-bimetal reagents was demonstrated by the high removals of DNAN, TOC and COD. Oxidation of the Mg-bimetal surface did not inhibit the treatment, as opposed to oxidation on ZVI or Fe-bimetals passivating the surface and inhibiting treatment. Future work may be performed on the impact of Distribution A: Approved for Public Release; Distribution is Unlimited 
537 catalytic metal dose relative to the base metal and the coating method during bimetal synthesis.

538 These parameters subsequently may be correlated to overall removals and removal kinetics. In

539 addition, the reaction occurred at neutral to basic $\mathrm{pH}$, i.e. without the need to lower the $\mathrm{pH}$ of the

540 treated solution as is commonly the case with Fe-based bimetals. This eliminates the need for an

541 additional chemical in a treatment scenario. Byproduct identification and subsequent

542 determination of the reaction pathways demonstrated that DNAN was reduced to amino

543 derivatives 2-ANAN, 4-ANAN and subsequently to DAAN. The 91\% closure for the carbon

544 mass balance indicated that the dissolved and adsorbed phases contained $12.4 \%$ and $78.4 \%$ TOC,

545 respectively. The significant carbon adsorbed to the Mg-bimetal surface requires further

546 investigation in either the extraction of these adsorbed compounds or direct analysis of used $\mathrm{Mg}$ -

547 bimetal particles. In both the dissolved and adsorbed phases, after complete product

548 identification, closure of the mass balance should be obtained. Furthermore, the proposed end-

549 product DAAN requires additional confirmation (i.e. evidence that further treatment of DAAN

550 does not form another compound).

551

552 Acknowledgements

553 This work was supported by the Consortium for Energy, Environment and Demilitarization

554 (CEED) contract number SINIT-15-0013. Electrospray ionization mass spectra were obtained in 555 the Center for Mass Spectrometry of Department of Chemistry and Chemical Biology of Stevens 556 Institute of Technology.

$558 \underline{\text { References }}$

559 Agarwal, S. et al. (2009) 'Reactivity of substituted chlorines and ensuing dechlorination 560 pathways of select PCB congeners with $\mathrm{Pd} / \mathrm{Mg}$ bimetallics', Environmental Science and

Distribution A: Approved for Public Release; Distribution is Unlimited 
561 Technology, 43(3), pp. 915-921. doi: 10.1021/es802538d.

562 Agarwal, S., Al-Abed, S. R. and Dionysiou, D. D. (2007) 'Enhanced corrosion-based Pd/Mg

563 bimetallic systems for dechlorination of PCBs', Environmental Science and Technology, 41(10),

564 pp. 3722-3727. doi: 10.1021/es062886y.

565 Agarwal, S., Al-Abed, S. R. and Dionysiou, D. D. (2009) 'Impact of organic solvents and 566 common anions on 2-chlorobiphenyl dechlorination kinetics with $\mathrm{Pd} / \mathrm{Mg}$, Applied Catalysis B:

567 Environmental, 92(1-2), pp. 17-22. doi: 10.1016/j.apcatb.2009.07.029.

568 Aginhotri, P., Mahidrakar, A. B. and Gautam, S. K. (2011) 'Complete dechlorination of

569 endosulfan and lindane using $\mathrm{Mg} 0 / \mathrm{Pd}+4$ bimetallic system', Water Environ. Res.,

570 83(September), pp. 865-873. doi: 10.2175/106143011X12928814445096.

571 Ahn, S. C. et al. (2011) 'Detoxification of PAX-21 ammunitions wastewater by zero-valent iron

572 for microbial reduction of perchlorate', Journal of Hazardous Materials, 192(2), pp. 909-914.

573 doi: 10.1016/j.jhazmat.2011.05.104.

574 Arthur, J. D. et al. (2017) 'Batch soil adsorption and column transport studies of 2,4-

575 dinitroanisole (DNAN) in soils', Journal of Contaminant Hydrology, 199, pp. 14-23. doi:

$576 \quad 10.1016 /$ j.jconhyd.2017.02.004.

577 Asgari, G., Ramavandi, B. and Farjadfard, S. (2013) 'Abatement of Azo Dye from Wastewater 578 Using Bimetal-Chitosan', 2013.

579 Begum, A. and Gautam, S. K. (2011) 'Dechlorination of endocrine disrupting chemicals using

$580 \quad \mathrm{Mg}^{0} / \mathrm{ZnCl}_{2}$ bimetallic system', Water Research. Elsevier Ltd, 45(7), pp. 2383-2391. doi:

$581 \quad 10.1016 /$ j.watres.2011.01.017.

582 Coutts, J. L. et al. (2011) 'The use of mechanical alloying for the preparation of palladized

583 magnesium bimetallic particles for the remediation of PCBs', Journal of Hazardous Materials. 
584 Elsevier B.V., 192(3), pp. 1380-1387. doi: 10.1016/j.jhazmat.2011.06.052.

585 Cwiertny, D. M. et al. (2006) 'Exploring the influence of granular iron additives on 1,1,1-

586 trichloroethane reduction', Environmental Science and Technology, 40(21), pp. 6837-6843. doi:

$587 \quad 10.1021 / \mathrm{es} 060921 \mathrm{v}$.

588 DeVor, R. et al. (2008) 'Dechlorination comparison of mono-substituted PCBs with $\mathrm{Mg} / \mathrm{Pd}$ in

589 different solvent systems', Chemosphere, 73(6), pp. 896-900. doi:

$590 \quad 10.1016 /$ j.chemosphere.2008.07.006.

591 DeVor, R. et al. (2009) 'Mechanism of the degradation of individual PCB congeners using

592 mechanically alloyed $\mathrm{Mg} / \mathrm{Pd}$ in methanol', Chemosphere. Elsevier Ltd, 76(6), pp. 761-766. doi:

593 10.1016/j.chemosphere.2009.05.007.

594 Fida, T. T. et al. (2014) 'Aerobic biodegradation of 2,4-dinitroanisole by Nocardioides sp. strain

595 JS1661', Applied and Environmental Microbiology, 80(24), pp. 7725-7731. doi:

596 10.1128/AEM.02752-14.

597 Fu, F., Cheng, Z. and Lu, J. (2015) 'Synthesis and use of bimetals and bimetal oxides in

598 contaminants removal from water: a review', $R S C A d v ., 5(104)$, pp. 85395-85409. doi:

$599 \quad 10.1039 /$ C5RA13067K.

600 Gautam, S. K. and Suresh, S. (2007) 'Complete dechlorination of DDE/DDD using

601 magnesium/palladium system', Water Environment Research, 79(Ddd), pp. 430-435. doi:

$60210.2175 / 106143006 \times 115336$.

603 Ghauch, A. and Tuqan, A. (2009) 'Reductive destruction and decontamination of aqueous

604 solutions of chlorinated antimicrobial agent using bimetallic systems', Journal of Hazardous

605 Materials, 164(2-3), pp. 665-674. doi: 10.1016/j.jhazmat.2008.08.048.

606 Hadnagy, E., Rauch, L. M. and Gardner, K. H. (2007) 'Dechlorination of polychlorinated

Distribution A: Approved for Public Release; Distribution is Unlimited 
607 biphenyls, naphthalenes and dibenzo-p-dioxins by magnesium/palladium bimetallic particles.', 608 Journal of environmental science and health. Part A, Toxic/hazardous substances \& 609 environmental engineering, 42(6), pp. 685-695. doi: 10.1080/10934520701326222.

610 Hawari, J. et al. (2015) 'Environmental fate of 2,4-dinitroanisole (DNAN) and its reduced 611 products', Chemosphere. Elsevier Ltd, 119, pp. 16-23. doi: 10.1016/j.chemosphere.2014.05.047.

612 Hudlicky, M. (Virginia P. I. and S. U. (1984) Reductions in Organic Chemistry - Hudlicky 613 M..pdf. John Wiley \& Sons.

614 Ileri, B., Ayyildiz, O. and Apaydin, O. (2015) 'Ultrasound-assisted activation of zero-valent 615 magnesium for nitrate denitrification: Identification of reaction by-products and pathways', 616 Journal of Hazardous Materials, 292(3), pp. 1-8. doi: 10.1016/j.jhazmat.2015.03.004.

617 Karthikeyan, S. and Spain, J. C. (2016) 'Biodegradation of 2,4-dinitroanisole (DNAN) by 618 Nocardioides sp. JS1661 in water, soil and bioreactors', Journal of Hazardous Materials.

619 Elsevier B.V., 312, pp. 37-44. doi: 10.1016/j.jhazmat.2016.03.029.

620 Khalil, A. M. E. et al. (2016) 'Promoting nitrate reduction kinetics by nanoscale zero valent iron 621 in water via copper salt addition', Chemical Engineering Journal. Elsevier B.V., 287, pp. 367622 380. doi: 10.1016/j.cej.2015.11.038.

623 Kitcher, E. et al. (2017) 'Characteristics and products of the reductive degradation of 3-nitro624 1,2,4-triazol-5-one (NTO) and 2,4-dinitroanisole (DNAN) in a Fe-Cu bimetal system', 625 Environmental Science and Pollution Research, 24(3), pp. 2744-2753. doi: 10.1007/s11356-016$626 \quad 8053-7$.

627 Koutsospyros, A. et al. (2012) 'Degradation of high energetic and insensitive munitions 628 compounds by Fe/Cu bimetal reduction', Journal of Hazardous Materials. Elsevier B.V., 219629 220, pp. 75-81. doi: 10.1016/j.jhazmat.2012.03.048. 
630 Lee, G. and Park, J. (2013) 'Reaction of zero-valent magnesium with water: Potential

631 applications in environmental remediation', Geochimica et Cosmochimica Acta. Elsevier Ltd,

632 102, pp. 162-174. doi: 10.1016/j.gca.2012.10.031.

633 Liu, J. et al. (2015) 'Selective removal of nitroaromatic compounds from wastewater in an 634 integrated zero valent iron (ZVI) reduction and ZVI/H2O2 oxidation process', RSC Advances.

635 Royal Society of Chemistry, 5, pp. 57444-57452. doi: 10.1039/C5RA08487C.

636 Luo, S. et al. (2010) 'Reductive degradation of tetrabromobisphenol A over iron-silver bimetallic

637 nanoparticles under ultrasound radiation', Chemosphere. Elsevier Ltd, 79(6), pp. 672-678. doi:

638 10.1016/j.chemosphere.2010.02.011.

639 Morales, J. et al. (2002) 'Hydrogenation of Phenol by the Pd/Mg and Pd/Fe Bimetallic Systems

640 under Mild Reaction Conditions', Industrial \& Engineering Chemistry Research, 41(13), pp.

641 3071-3074. doi: 10.1021/ie0200510.

642 Nie, X. et al. (2013) 'Rapid degradation of hexachlorobenzene by micron Ag/Fe bimetal

643 particles', Journal of Environmental Sciences (China). The Research Centre for Eco-

644 Environmental Sciences, Chinese Academy of Sciences, 25(3), pp. 473-478. doi:

645 10.1016/S1001-0742(12)60088-6.

646 Patel, U. D. and Suresh, S. (2007) 'Dechlorination of chlorophenols using magnesium-palladium

647 bimetallic system', Journal of Hazardous Materials, 147(1-2), pp. 431-438. doi:

$648 \quad$ 10.1016/j.jhazmat.2007.01.029.

649 Patel, U. D. and Suresh, S. (2008) 'Effects of solvent, pH, salts and resin fatty acids on the

650 dechlorination of pentachlorophenol using magnesium-silver and magnesium-palladium

651 bimetallic systems', Journal of Hazardous Materials, 156(1-3), pp. 308-316. doi:

652 10.1016/j.jhazmat.2007.12.021.

Distribution A: Approved for Public Release; Distribution is Unlimited 
653 Ramavandi, B. et al. (2011) 'Experimental investigation of the chemical reduction of nitrate ion

654 in aqueous solution by $\mathrm{Mg} / \mathrm{Cu}$ bimetallic particles', Reaction Kinetics, Mechanisms and

655 Catalysis, 102(2), pp. 313-329. doi: 10.1007/s11144-010-0274-z.

656 Rao, B. et al. (2013a) 'Photochemical transformation of the insensitive munitions compound 2,4-

657 dinitroanisole', Science of the Total Environment, 443, pp. 692-699. doi:

$658 \quad$ 10.1016/j.scitotenv.2012.11.033.

659 Rao, B. et al. (2013b) 'Photochemical transformation of the insensitive munitions compound 2,4-

660 dinitroanisole', Science of the Total Environment. Elsevier B.V., 443, pp. 692-699. doi:

$661 \quad$ 10.1016/j.scitotenv.2012.11.033.

662 Rivero-Huguet, M. and Marshall, W. D. (2009) 'Reduction of hexavalent chromium mediated by 663 micro- and nano-sized mixed metallic particles', Journal of Hazardous Materials, 169(1-3), pp.

664 1081-1087. doi: 10.1016/j.jhazmat.2009.04.062.

665 Saitta, E. K. H. et al. (2015) 'Case study of a non-destructive treatment method for the 666 remediation of military structures containing polychlorinated biphenyl contaminated paint',

667 Journal of Environmental Management. Elsevier Ltd, 158, pp. 40-47. doi:

$668 \quad$ 10.1016/j.jenvman.2015.04.038.

669 Shen, J. et al. (2013) 'Pretreatment of 2, 4-dinitroanisole ( DNAN ) producing wastewater using 670 a combined zero-valent iron ( ZVI ) reduction and Fenton oxidation process', Journal of 671 Hazardous Materials, 260, pp. 993-1000. doi: 10.1016/j.jhazmat.2013.07.003.

672 Shih, Y. hsin et al. (2009) 'Dechlorination of hexachlorobenzene by using nanoscale Fe and 673 nanoscale Pd/Fe bimetallic particles', Colloids and Surfaces A: Physicochemical and

674 Engineering Aspects, 332(2-3), pp. 84-89. doi: 10.1016/j.colsurfa.2008.09.031.

675 Solanki, J. N. and Murthy, Z. V. P. (2011) 'Reduction of 4-chlorophenol by Mg and Mg-Ag 
676 bimetallic nanocatalysts', Industrial and Engineering Chemistry Research, 50(24), pp. 14211677 14216. doi: 10.1021/ie2022338.

678 Sun, Y. et al. (2016) 'The influences of iron characteristics, operating conditions and solution 679 chemistry on contaminants removal by zero-valent iron: A review', Water Research, 100, pp. 680 277-295. doi: 10.1016/j.watres.2016.05.031.

681 Taylor, S. et al. (2017) 'Photo-degradation of 2,4-dinitroanisole (DNAN): An emerging 682 munitions compound', Chemosphere, 167, pp. 193-203. doi:

683 10.1016/j.chemosphere.2016.09.142.

684 Xiong, Z. et al. (2015) 'Comparative study on the reactivity of Fe/Cu bimetallic particles and 685 zero valent iron (ZVI) under different conditions of $\mathrm{N}<\inf >2</ \mathrm{inf}>$, air or without aeration', 686 Journal of Hazardous Materials. Elsevier B.V., 297, pp. 261-268. doi:

$687 \quad$ 10.1016/j.jhazmat.2015.05.006.

$688 \mathrm{Xu}, \mathrm{F}$. et al. (2012) 'Highly active and stable Ni-Fe bimetal prepared by ball milling for catalytic 689 hydrodechlorination of 4-chlorophenol', Environmental Science and Technology, 46(8), pp. 690 4576-4582. doi: 10.1021/es203876e.

$691 \mathrm{Xu}, \mathrm{X}$. et al. (2005) 'Catalytic dechlorination kinetics of p-dichlorobenzene over Pd/Fe 692 catalysts', Chemosphere, 58(8), pp. 1135-1140. doi: 10.1016/j.chemosphere.2004.07.010. 
694

695
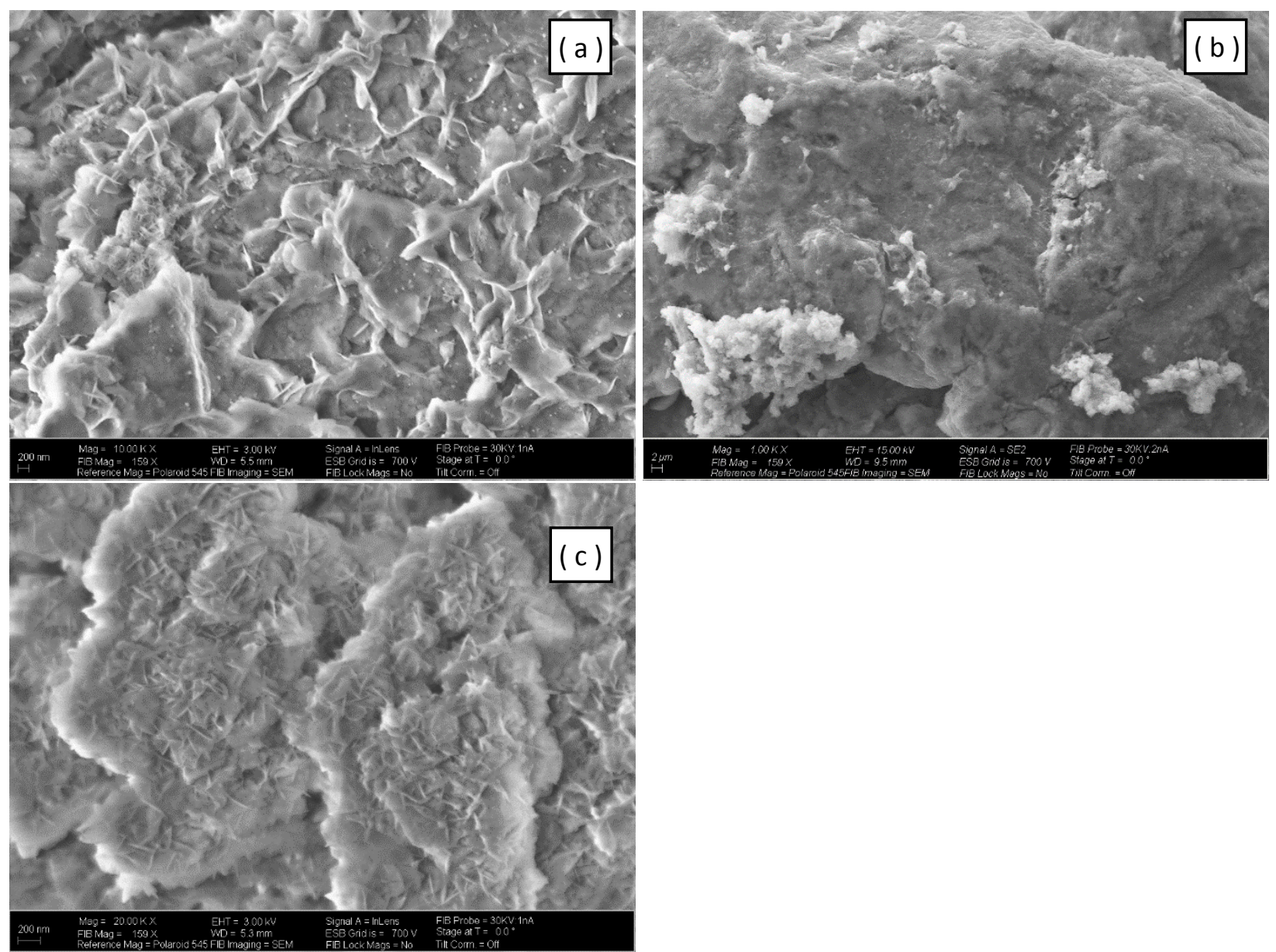

696

697

$698 \quad$ Fig 1

699 Images output by SEM Analysis.

700

701

Distribution A: Approved for Public Release; Distribution is Unlimited 
702

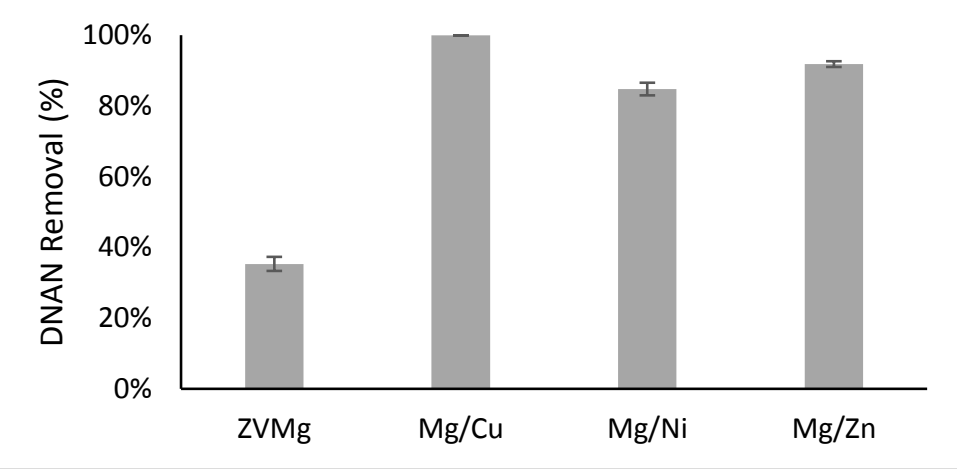

$703 \quad$ Fig 2

704 Created on Microsoft Excel.

705

706

707

Distribution A: Approved for Public Release; Distribution is Unlimited 
708

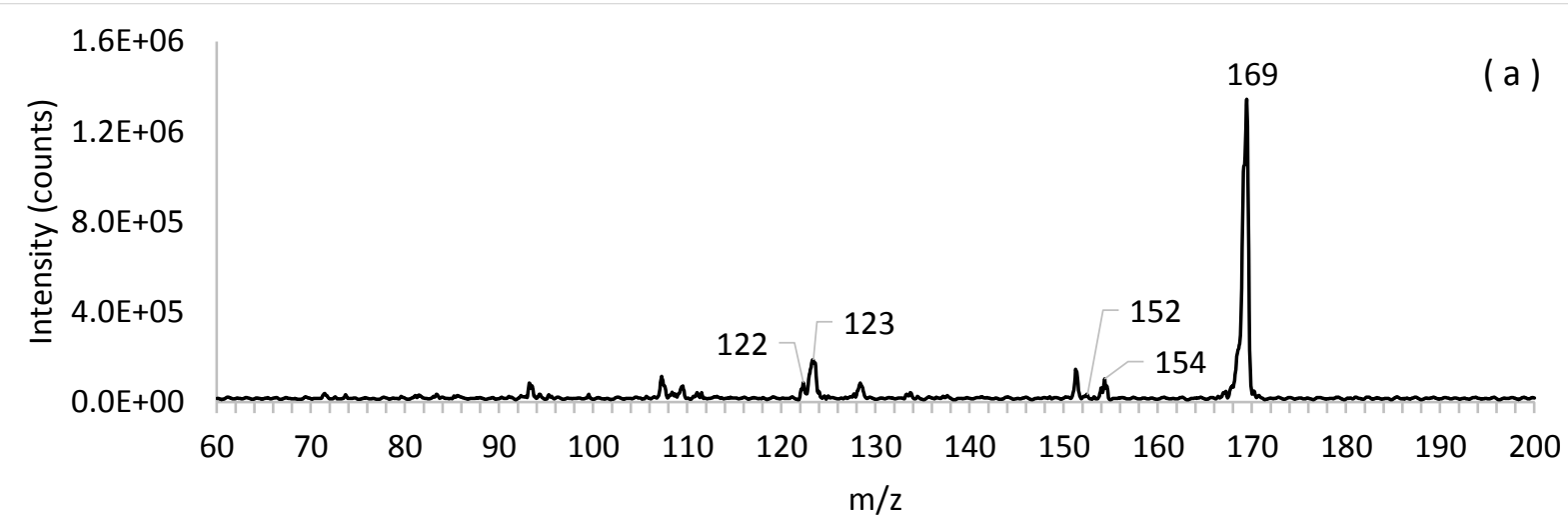

709
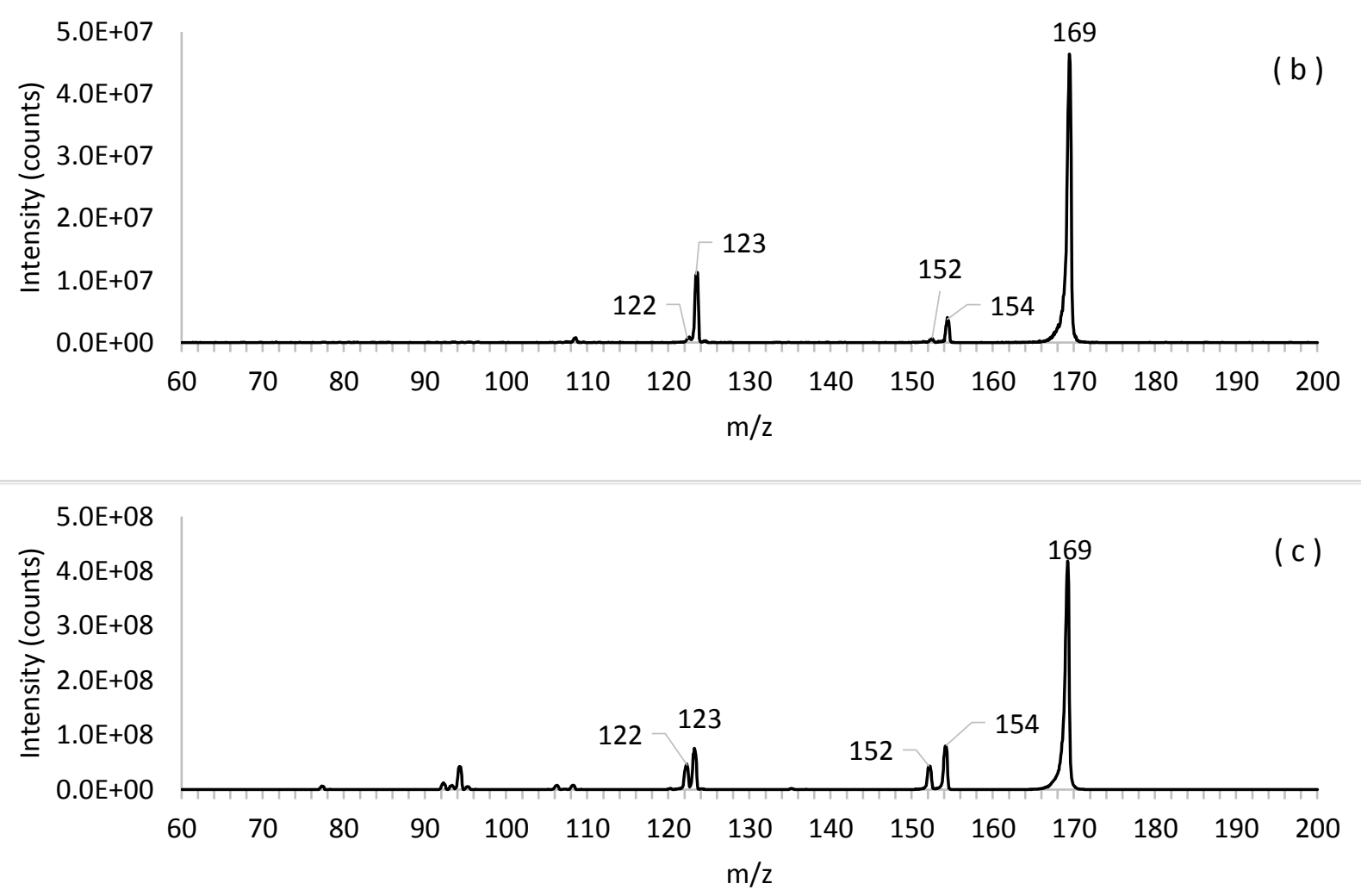

711

$712 \quad$ Fig 3

713 Figure generated by Microsoft Excel.

714

715

Distribution A: Approved for Public Release; Distribution is Unlimited 


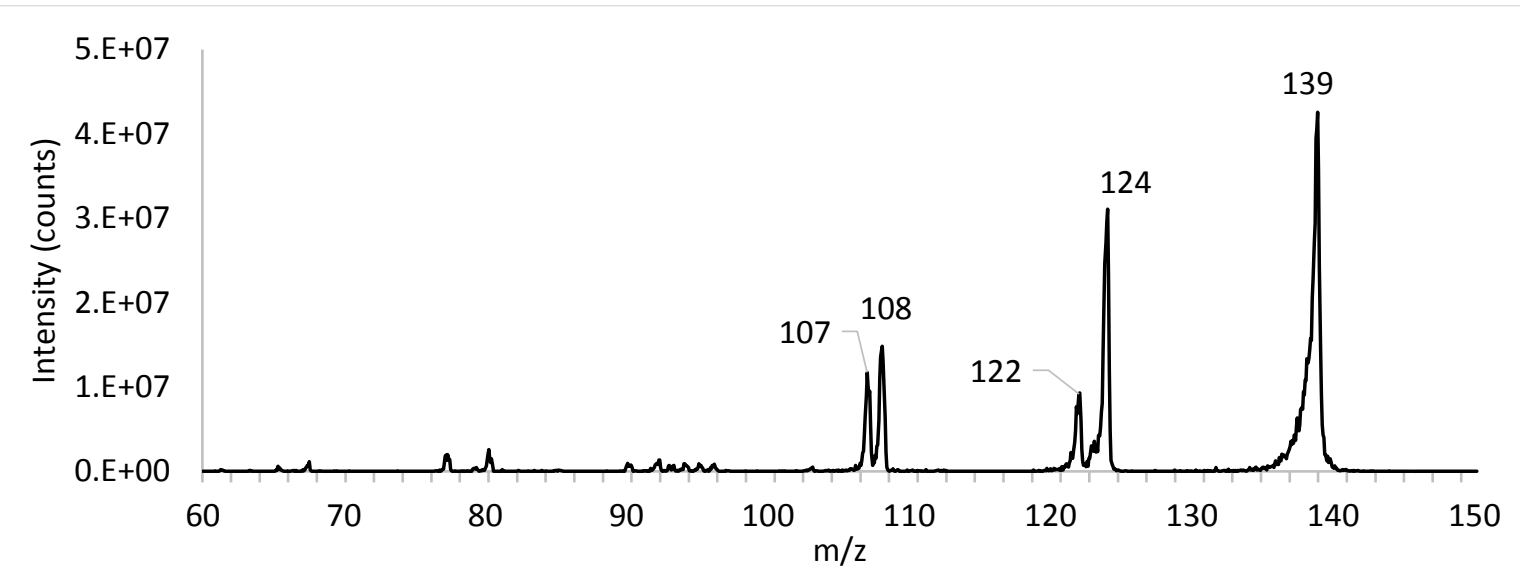

$717 \quad$ Fig 4

718 Figure generated by Microsoft Excel.

719

720

Distribution A: Approved for Public Release; Distribution is Unlimited 
721

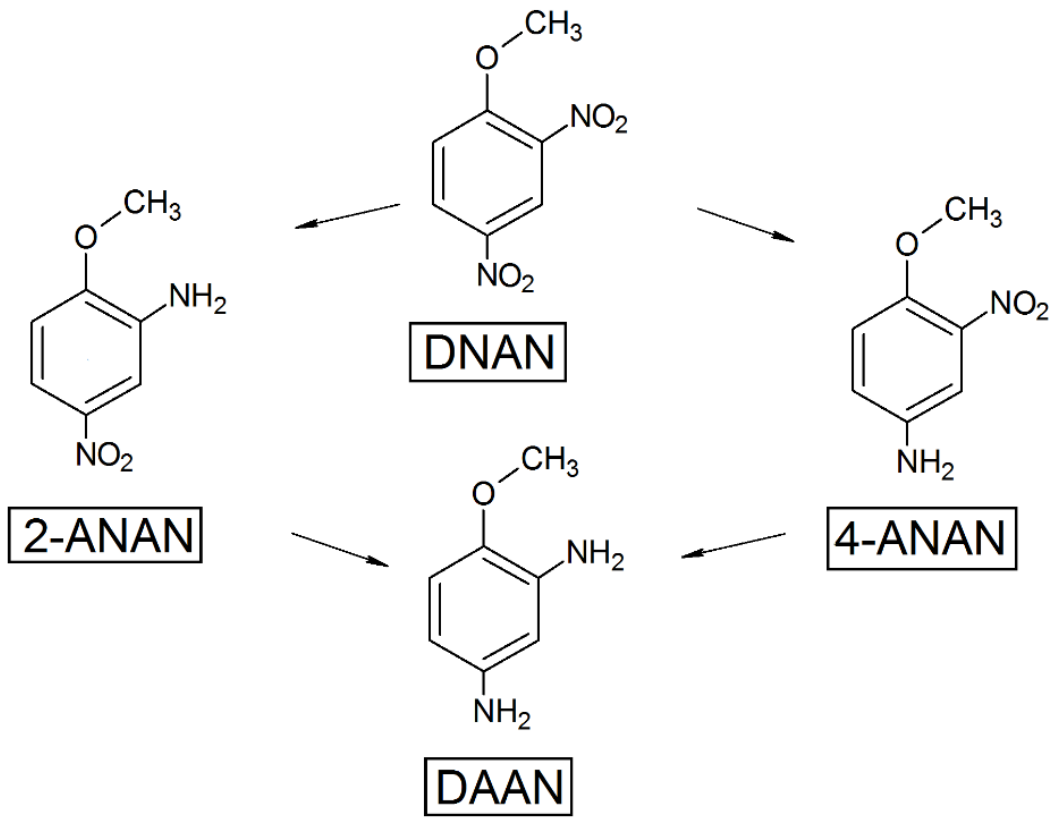

722

$723 \quad$ Fig 5

724 Figure generated by ChemCad Free Version.

725

726 


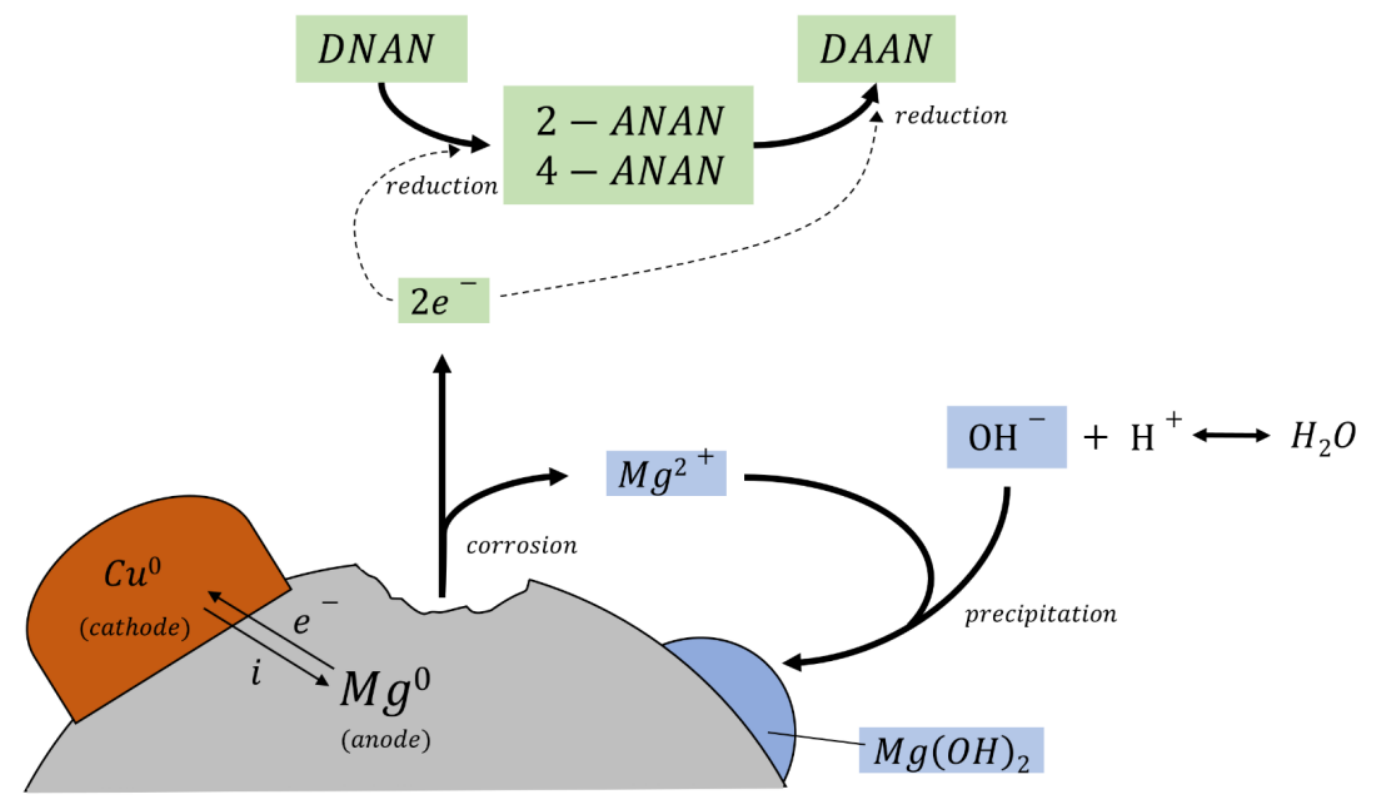

727

$728 \quad$ Fig 6

729 Generated on Microsoft Powerpoint

730

731

Distribution A: Approved for Public Release; Distribution is Unlimited 


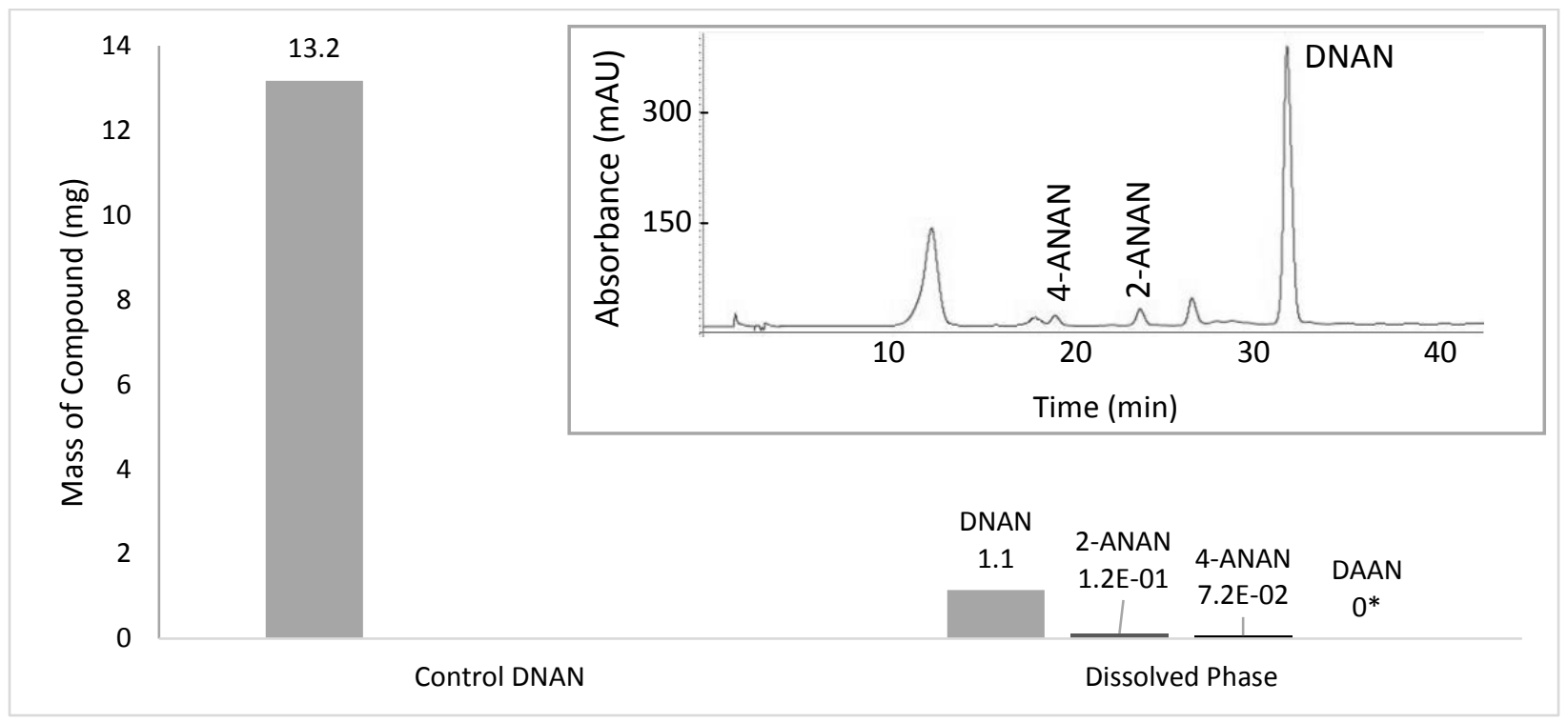

734

735

Fig 7

736 Figure generated by Microsoft Excel and Word.

737

738

Distribution A: Approved for Public Release; Distribution is Unlimited 


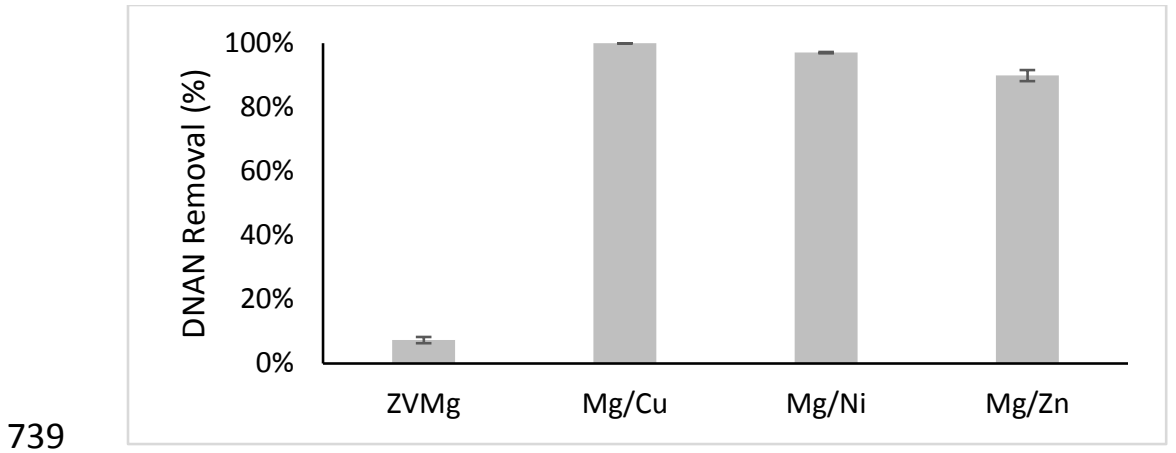

$740 \quad$ Fig 8

741 Created on Microsoft Excel.

742

743

744

Distribution A: Approved for Public Release; Distribution is Unlimited 


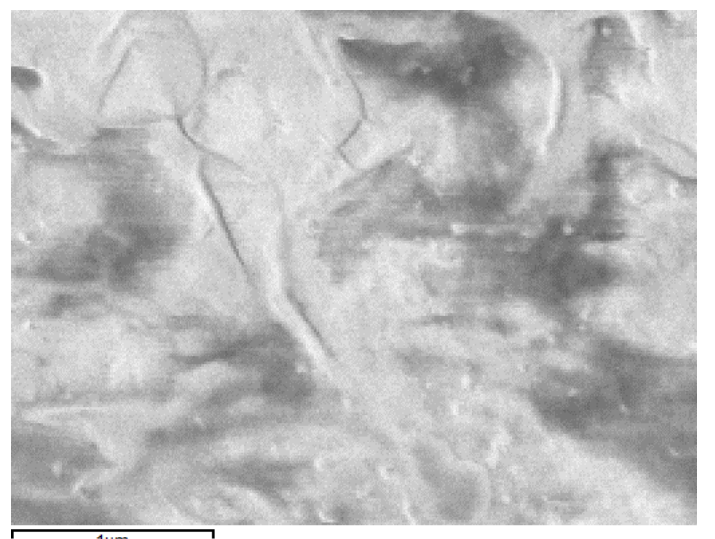

( a )

745

SEM: $\mathrm{Mg} / \mathrm{Cu}$

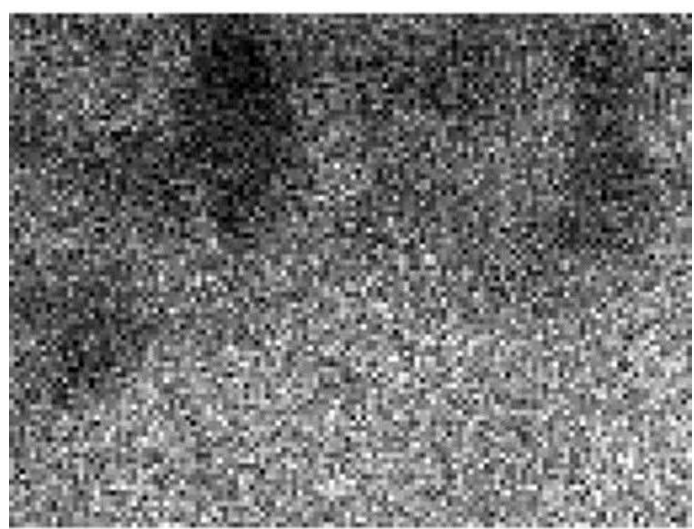

746

0

(c)

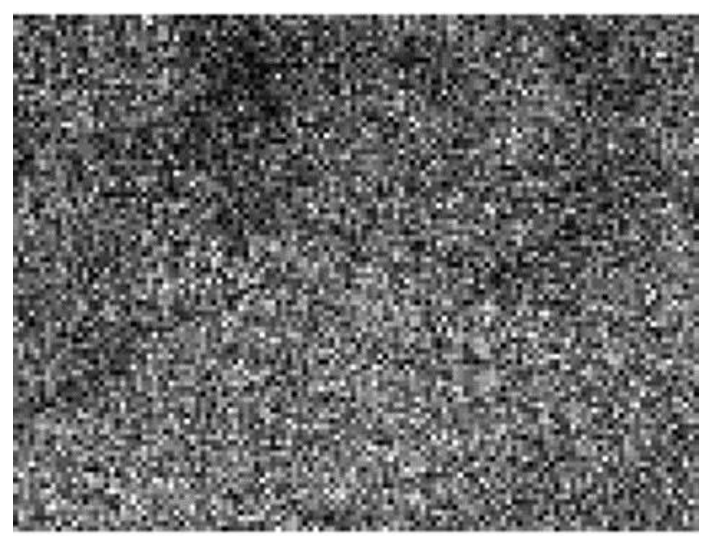

$\mathrm{Mg}$

( b )

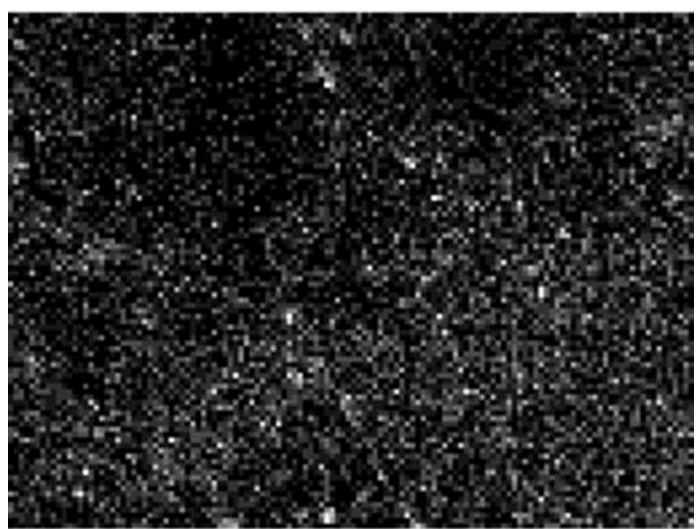

$\mathrm{Cu}$

(d)

$747 \quad$ Fig 9

748 Images generated by EDS mapping. 


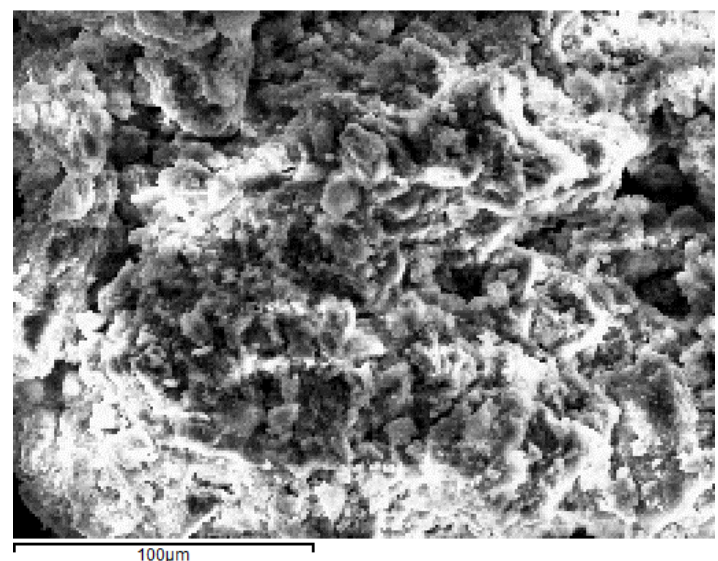

751

SEM: $\mathrm{Mg} / \mathrm{Cu}$

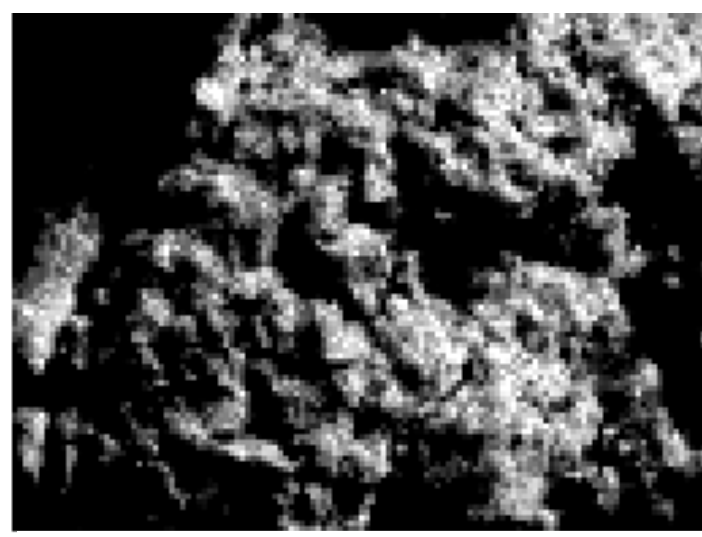

$\mathrm{O}$

(c)

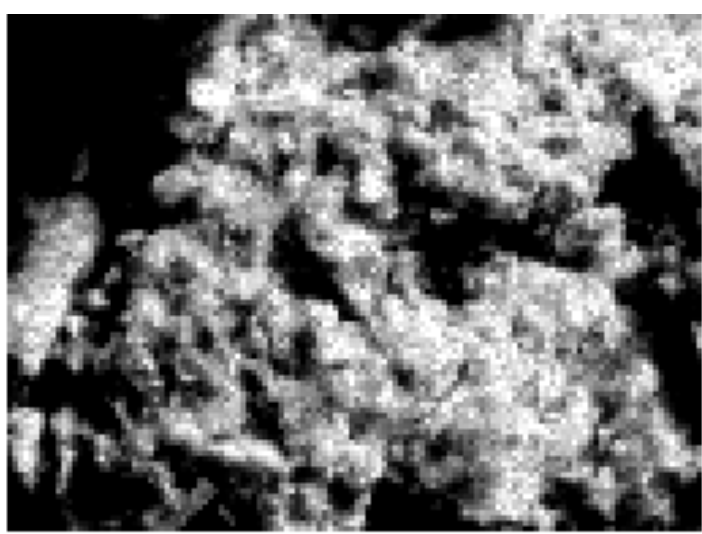

(b )

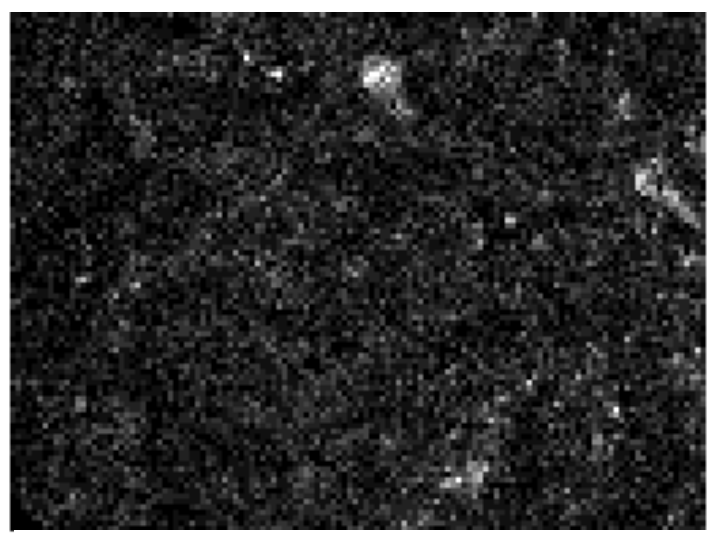

$\mathrm{Cu}$

(d)

752

Fig 10

754

Images generated by EDS mapping. 
757

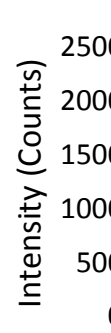

(a)

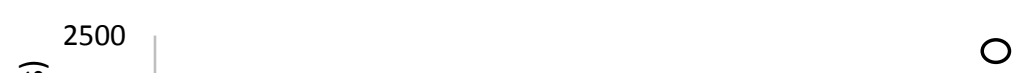

(b)

758

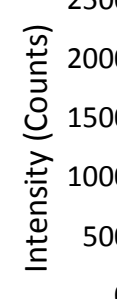

0
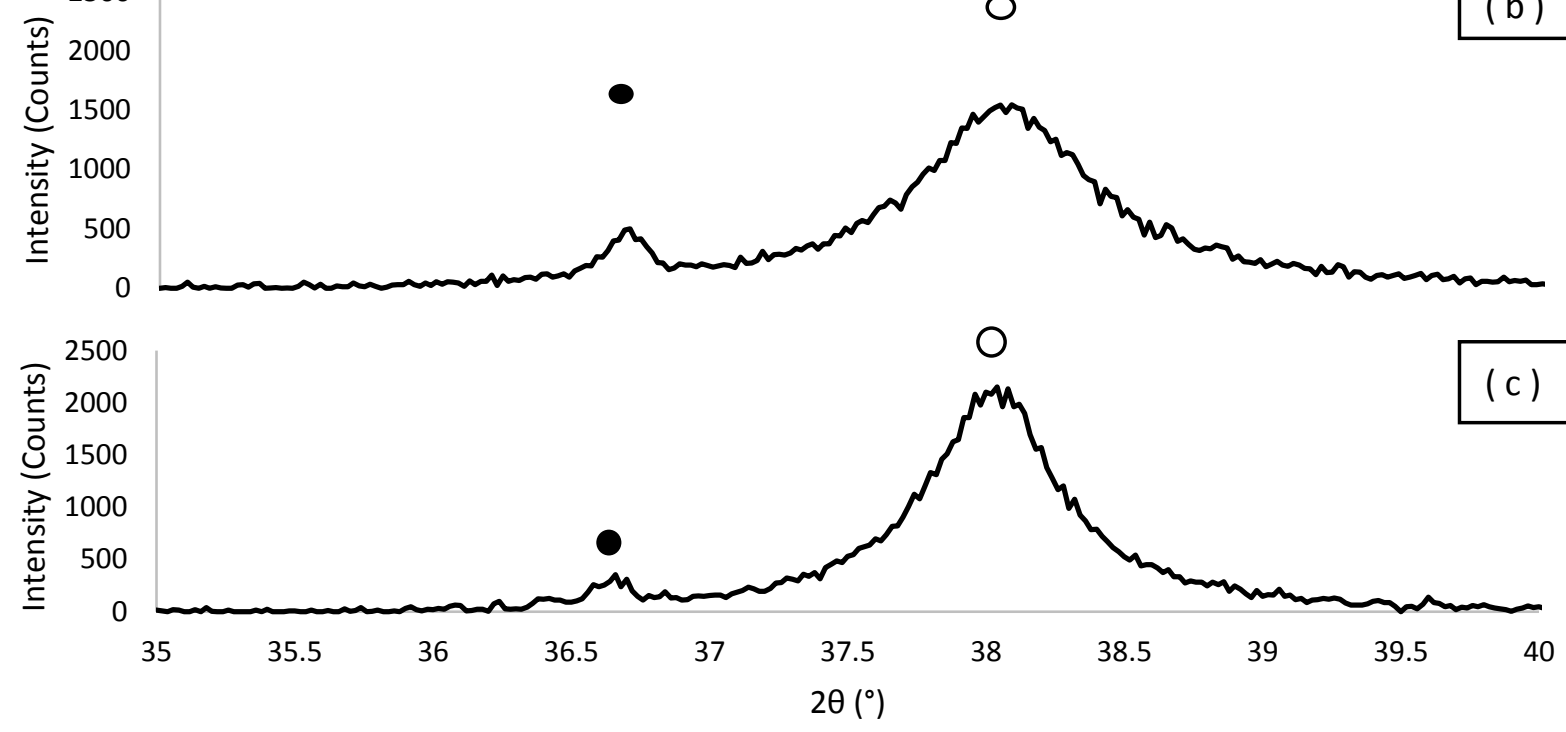

759

$760 \quad$ Fig 11

761 Figure generated by Microsoft Excel.

762 


\title{
Supplemental Information
}

\section{Characterization of Mg-based Bimetal Treatment of Insensitive Munition 2,4- dinitroanisole}

\author{
Emese Hadnagy ${ }^{1, *}$, Andrew Mai $^{2}$, Benjamin Smolinski ${ }^{3}$, Washington Braida ${ }^{2}$, Agamemnon \\ Koutsospyros $^{1}$
}

Submitted to:

Environmental Science and Pollution Research

*Corresponding Author. Email address: EHadnagy@newhaven.edu ${ }^{1}$ Department of Civil and Environmental Engineering, University of New Haven ${ }^{2}$ Department of Civil, Environmental, and Ocean Engineering, Stevens Institute of Technology ${ }^{3}$ RDECOM-ARDEC

Distribution A: Approved for Public Release; Distribution is Unlimited 
Table S.1 Elemental Compositions (\% Mass) of Bimetal Surfaces

\begin{tabular}{|c|c|c|c|c|}
\hline Bimetal & & O & Mg & $\begin{array}{c}\mathbf{C u}, \mathbf{~ N i} \\
\text { or Zn }\end{array}$ \\
\hline $\mathrm{Mg} / \mathrm{Cu}$ & Unused & 53.1 & 34.7 & 12.2 \\
\hline & Used & 65.6 & 34.4 & 0.6 \\
\hline $\mathrm{Mg} / \mathrm{Ni}$ & Unused & 44.7 & 14.7 & 40.6 \\
\hline & Used & 54.4 & 18.8 & 26.8 \\
\hline $\mathrm{Mg} / \mathrm{Zn}$ & Unused & 41.9 & 23.4 & 34.7 \\
\hline & Used & 53.8 & 31.5 & 14.7 \\
\hline
\end{tabular}

Distribution A: Approved for Public Release; Distribution is Unlimited 


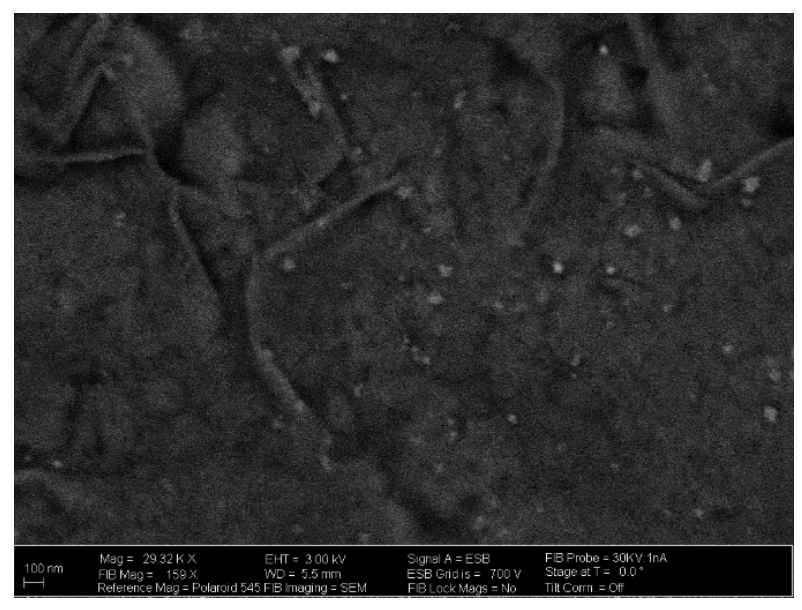

Fig. S.1 Contrasted SEM image of sample surface of unused $\mathrm{Mg} / \mathrm{Cu}$ to more easily observe $\mathrm{Cu}$ nanoparticles 

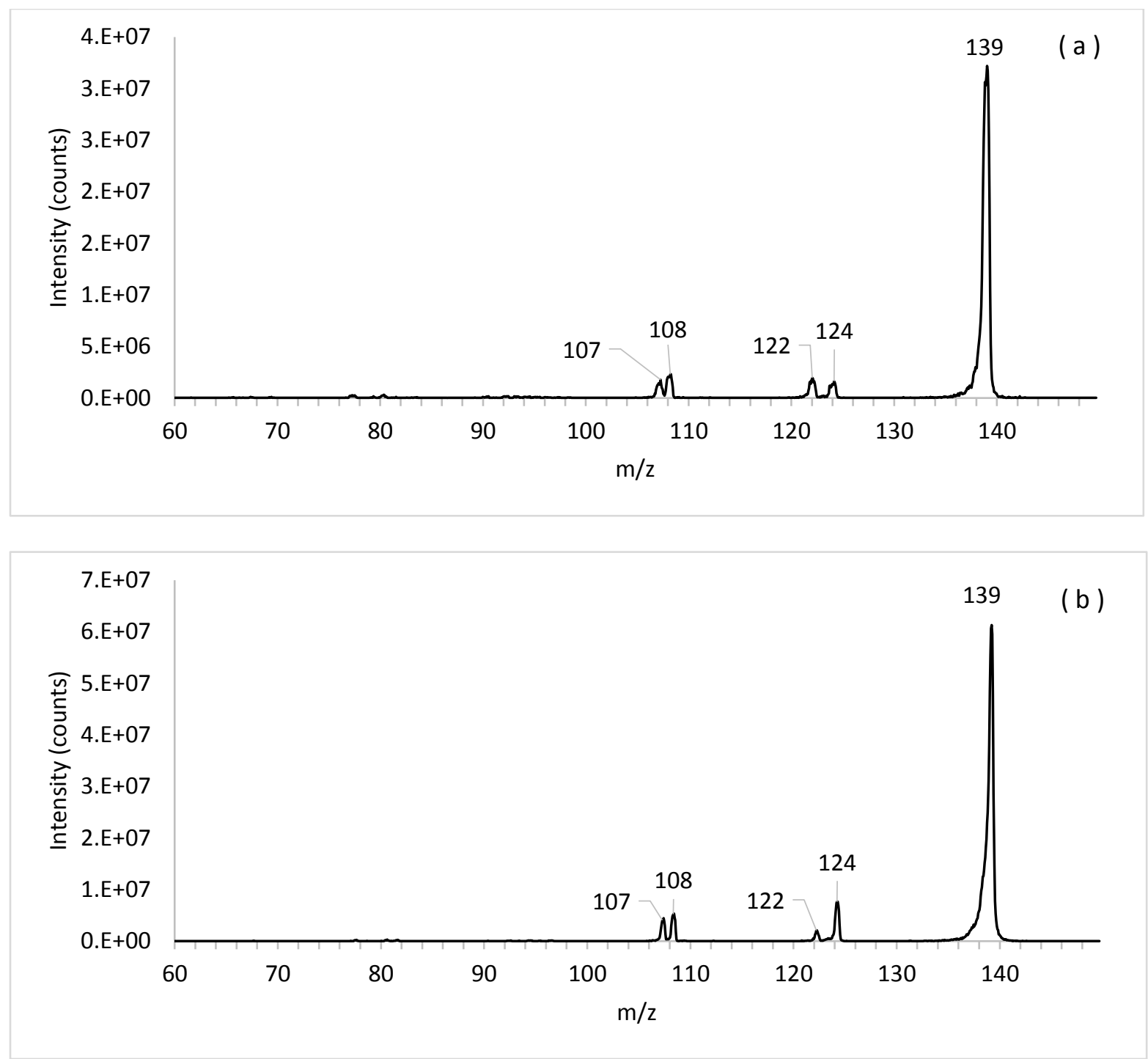

Fig. S.2 Daughter spectrum of m/z 139 from ESI-MS/MS in positive mode from (a) after DNAN treatment (solvent matrix, $0.5 \% \mathrm{~S} / \mathrm{L}, 10: 1 \mathrm{Mg}$ to $\mathrm{Cu}$ ratio and $2.5 \mathrm{hr}$ treatment) and (b) pure DAAN solution reference

Distribution A: Approved for Public Release; Distribution is Unlimited 


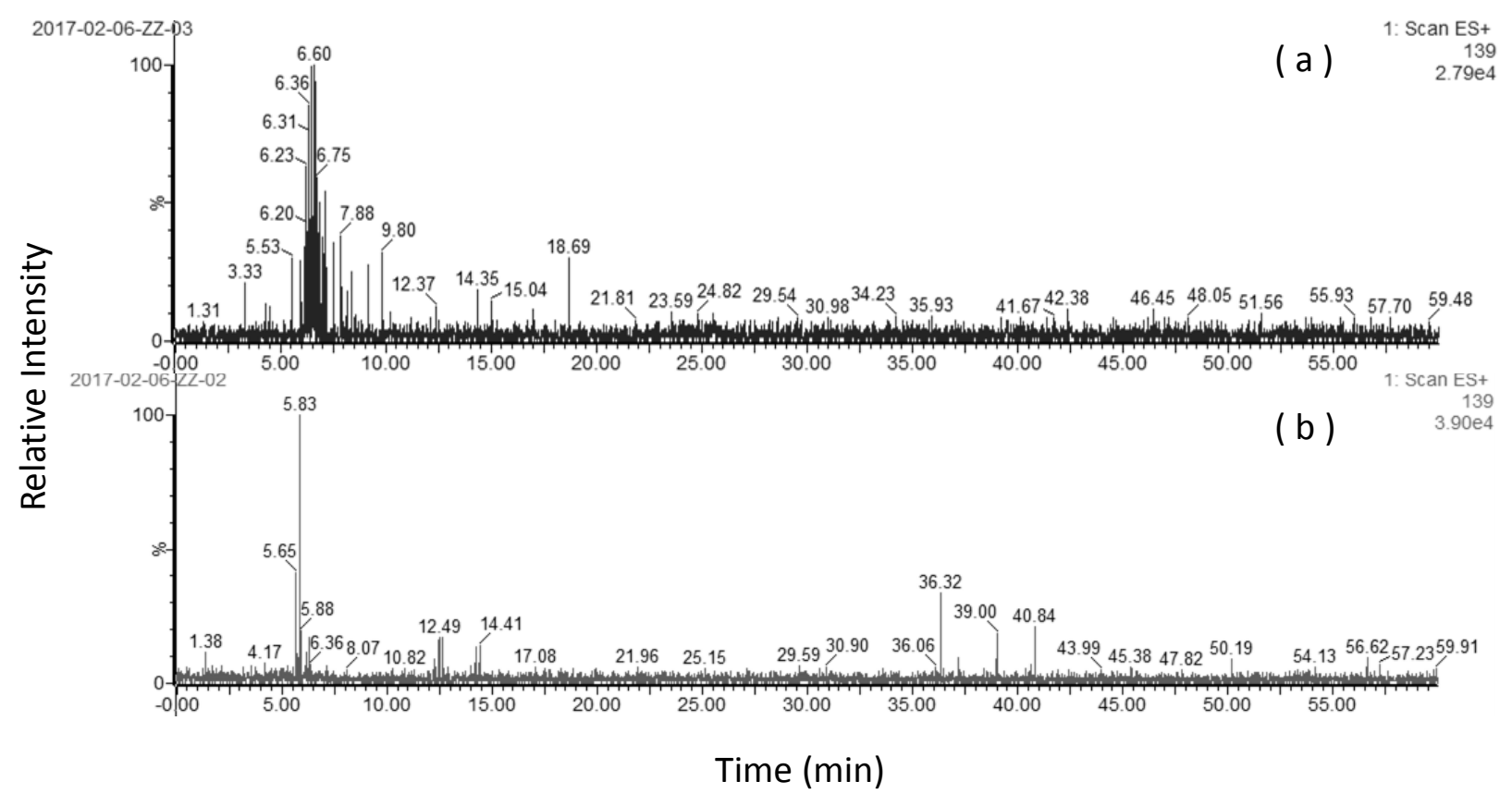

Fig. S. 3 Mass chromatograms of selected ion m/z 139 obtained from HPLC-ESI-MS of (a) pure DAAN, and (b) treated 4-ANAN sample (aqueous solution, $0.5 \% \mathrm{~S} / \mathrm{L}, 10: 1 \mathrm{Mg}$ to $\mathrm{Cu}$ ratio and 1 $\mathrm{hr}$ treatment) where the elution of $\mathrm{m} / \mathrm{z} 139$ was identical. The slight difference in elution times $(<1 \mathrm{~min})$ was due to peak shifts on HPLC 


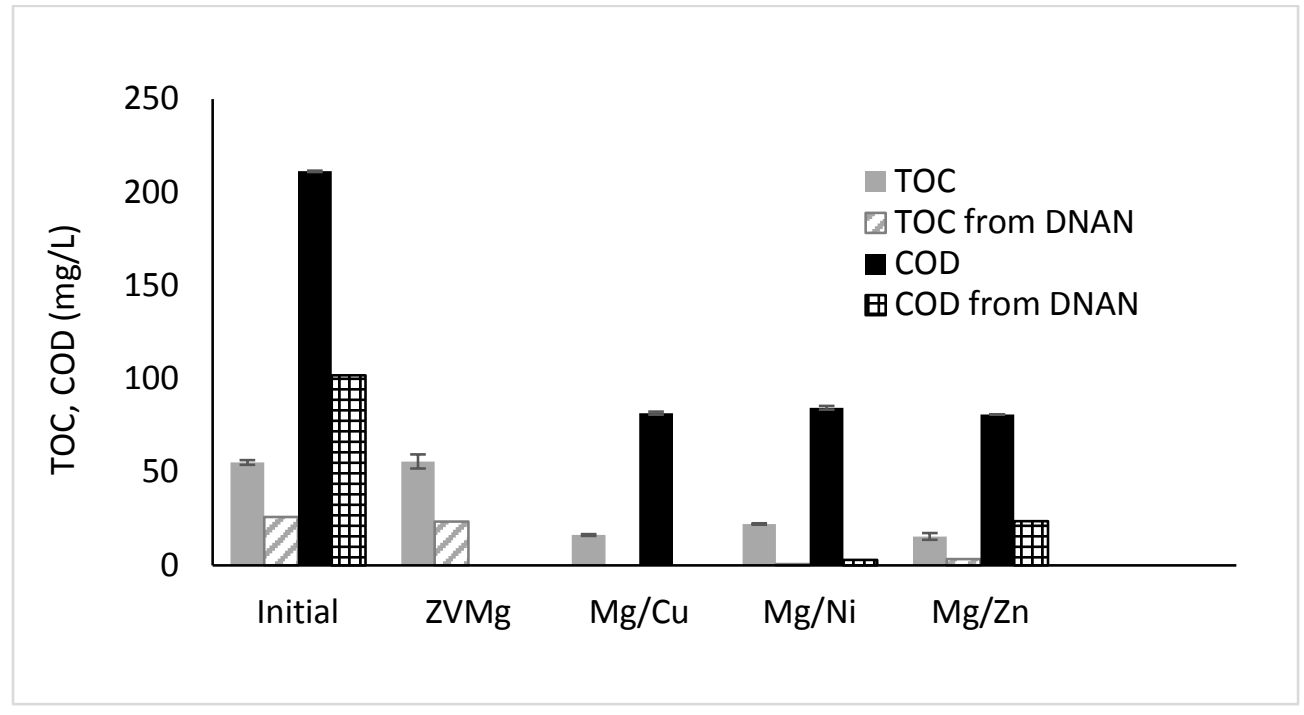

Fig. S.4 TOC, COD $\left(\mathrm{mg} \mathrm{L}^{-1}\right)$ and DNAN contribution to TOC and COD in treated wastewater $(0.5 \% \mathrm{~S} / \mathrm{L}, 10: 1 \mathrm{Mg}$ to catalytic metal ratio, and $2.5 \mathrm{~h}$ treatment time, COD not measured for ZVMg) 


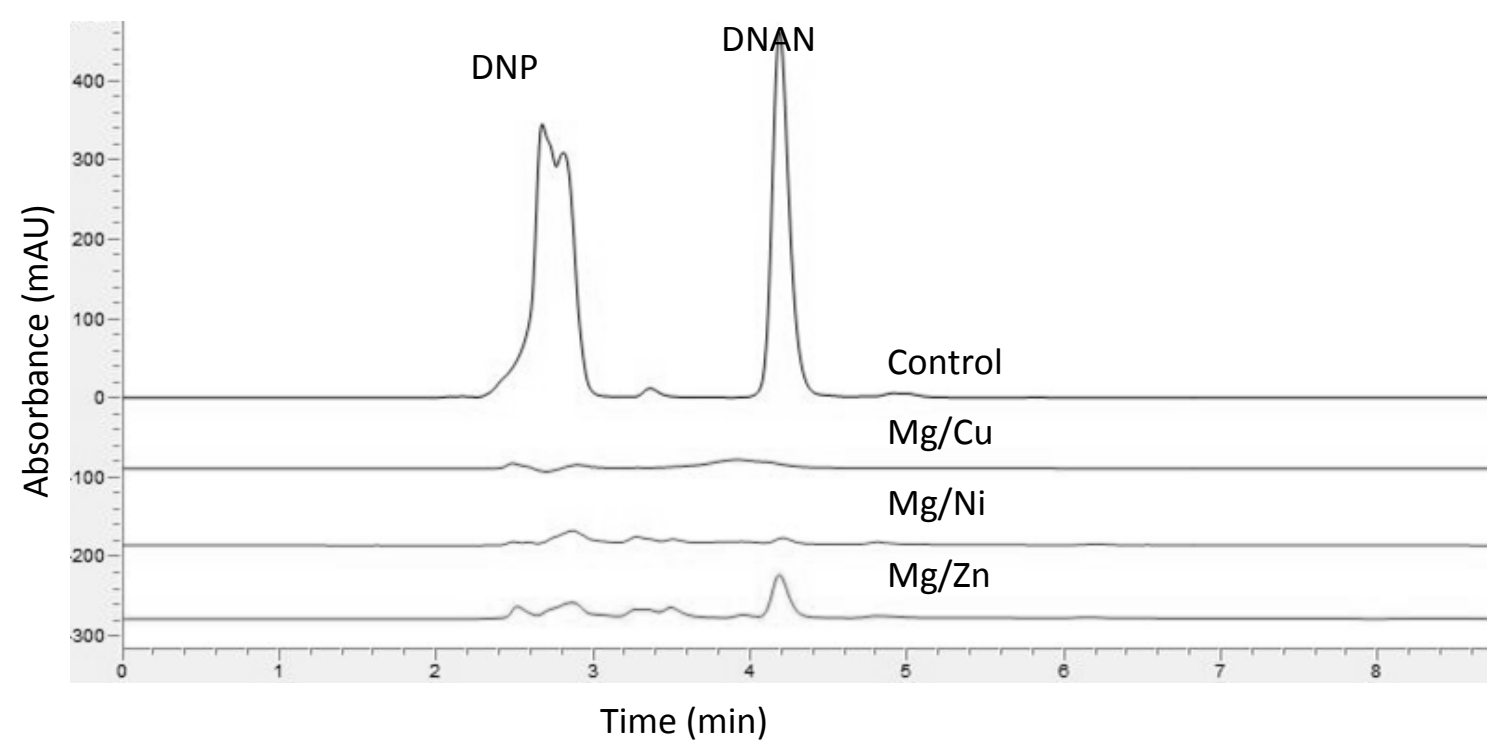

Fig. S.5 Visualization of degradation of DNP (2.1 min) and DNAN (4.2 min) in the wastewater control (top chromatogram) versus wastewater treated with $\mathrm{Mg} / \mathrm{Cu}, \mathrm{Mg} / \mathrm{Ni}$ and $\mathrm{Mg} / \mathrm{Zn}$ using overlaid chromatograms after 150 minutes of treatment (wastewater matrix, $0.5 \% \mathrm{~S} / \mathrm{L}, 10: 1 \mathrm{Mg}$ to secondary metal ratio) 


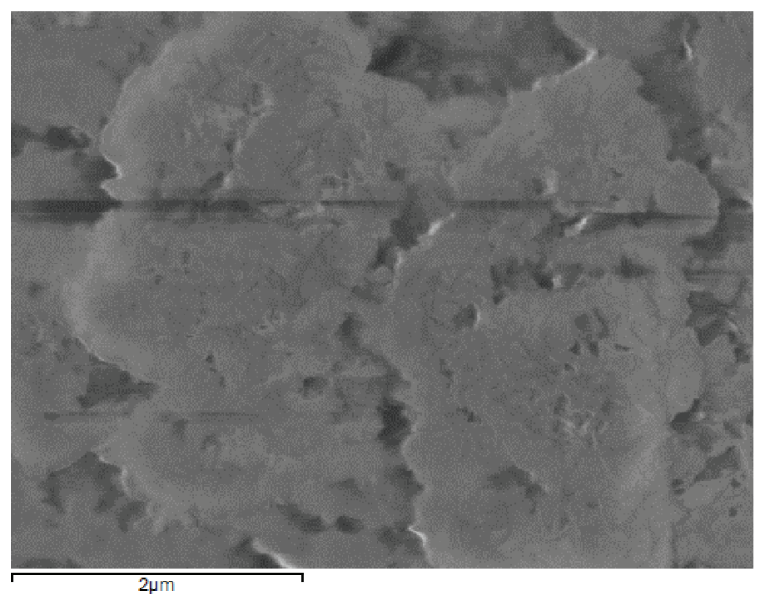

SEM: $\mathrm{Mg} / \mathrm{Ni}$

( a )

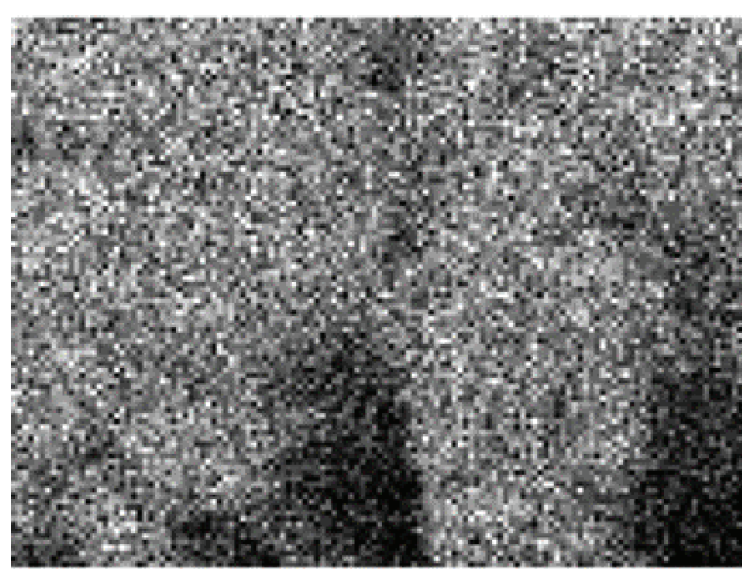

$\mathrm{O}$

(c)

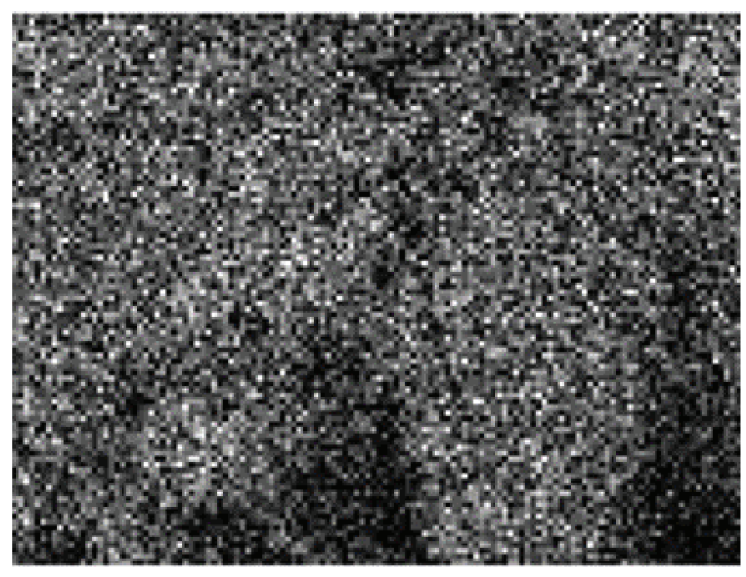

$\mathrm{Mg}$

( b )

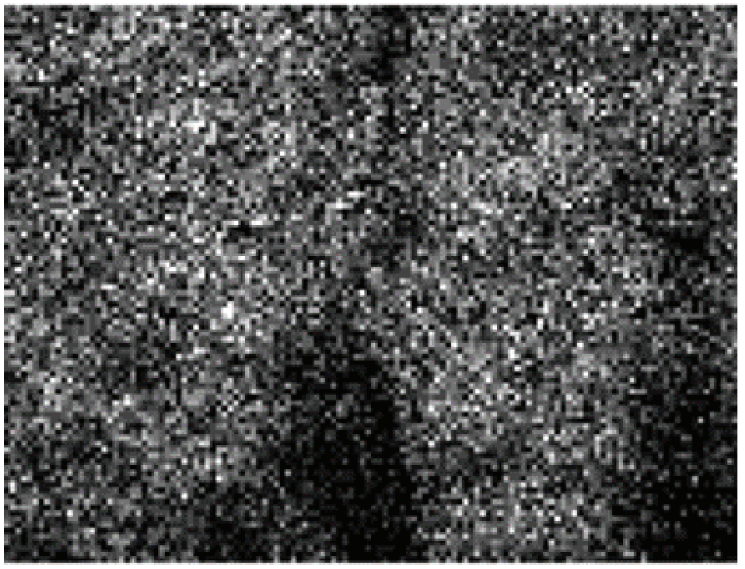

$\mathrm{Ni}$

(d)

Fig. S.6 EDS mapping of (a) sample region of an unused particle of $\mathrm{Mg} / \mathrm{Ni}$ pictured by SEM elucidating distribution of (b) primary metal $\mathrm{Mg}$ to (c) oxygen, and (d) catalytic metal $\mathrm{Ni}$ 


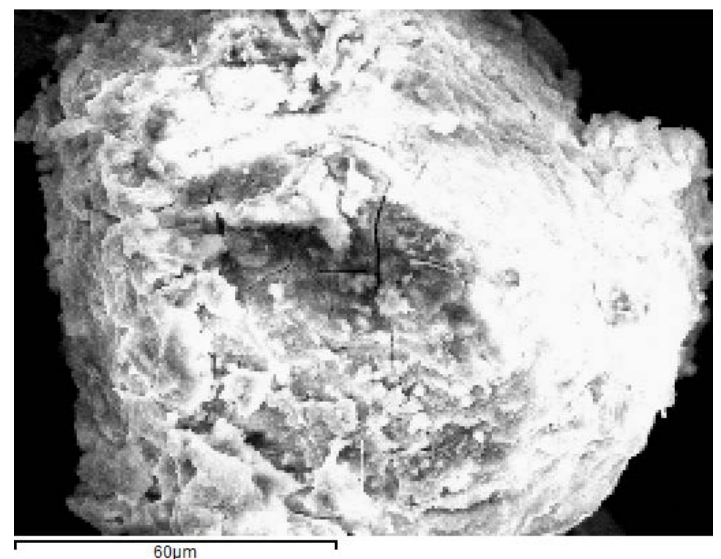

SEM: $\mathrm{Mg} / \mathrm{Ni}$

( a )

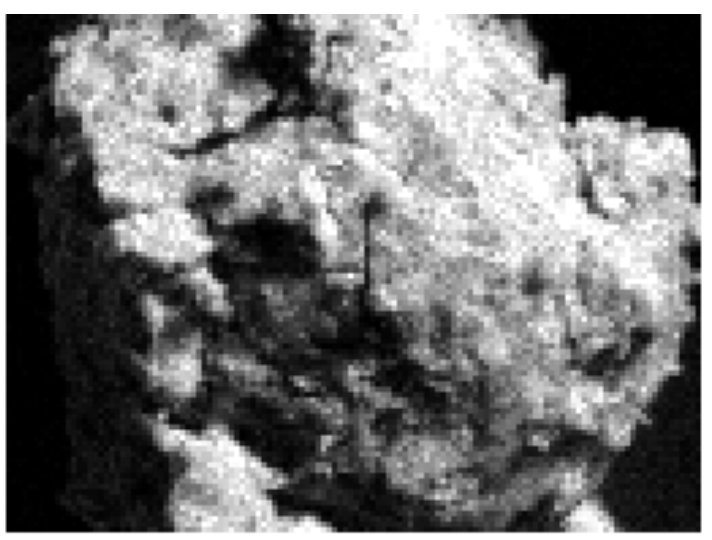

0

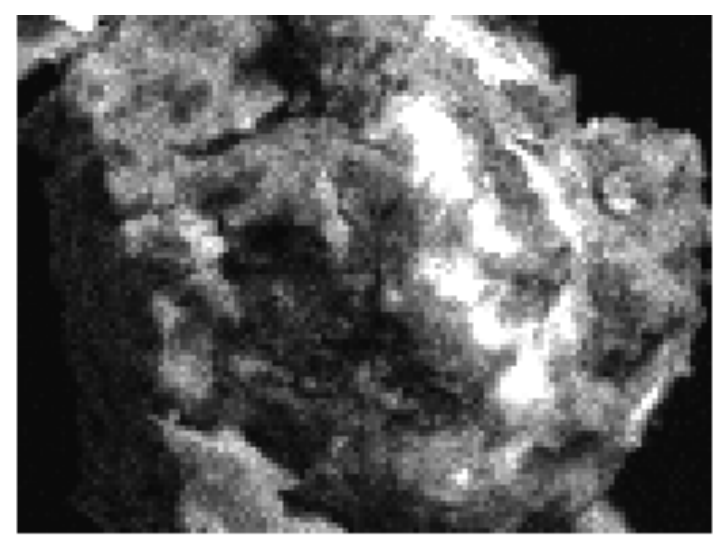

Mg

( b )

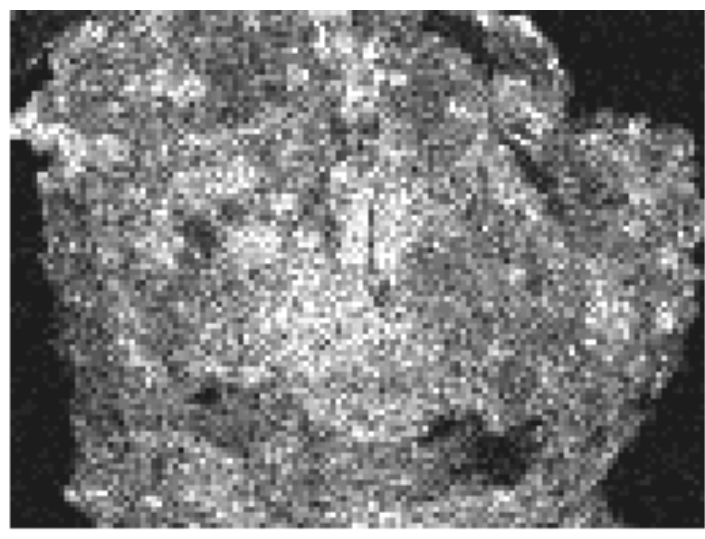

$\mathrm{Ni}$

(d)

Fig. S.7 EDS mapping of (a) sample region of a used particle of Mg/Ni pictured by SEM elucidating distribution of (b) primary metal $\mathrm{Mg}$ to (c) oxygen, and (d) catalytic metal $\mathrm{Ni}$ 

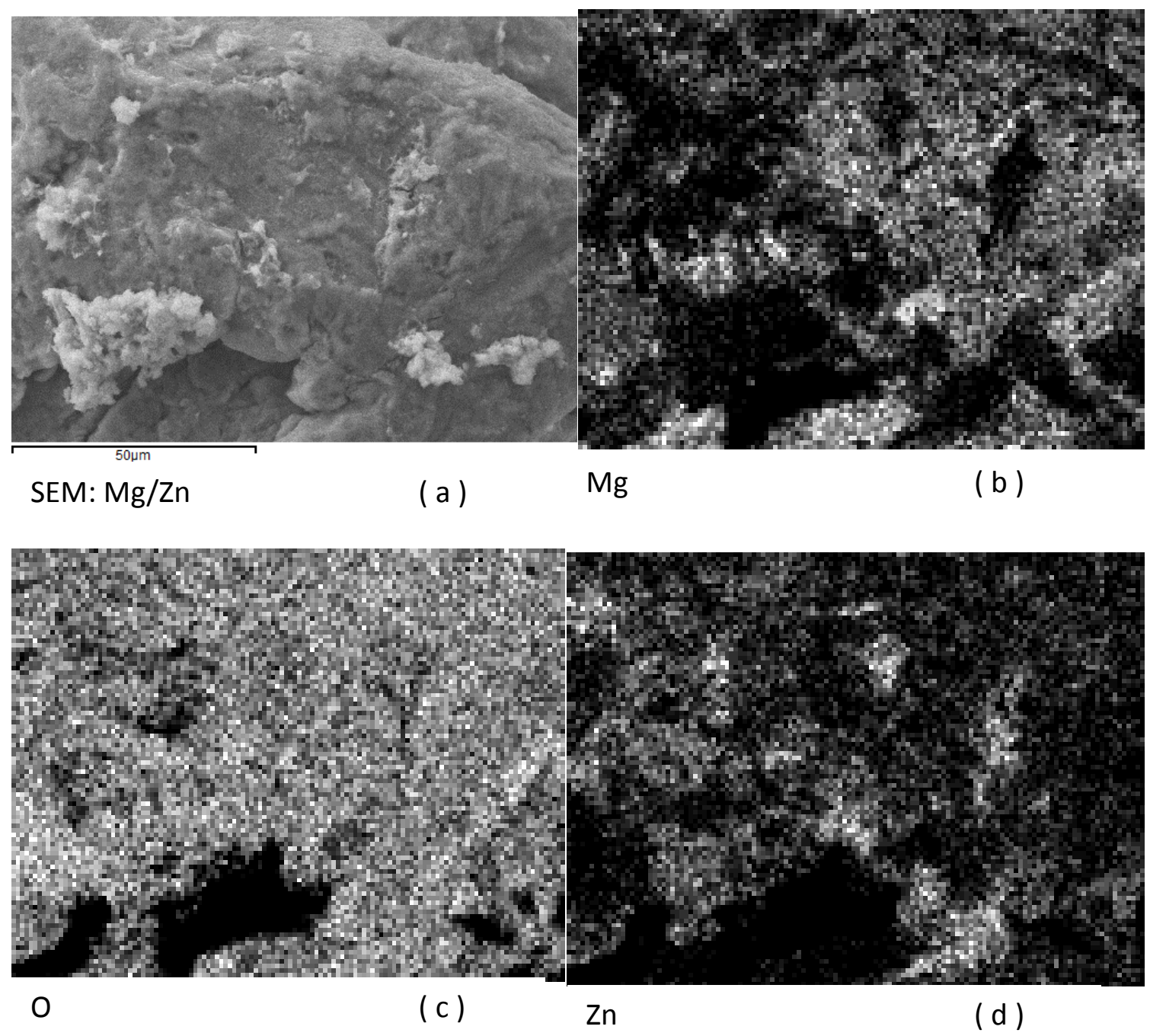

Fig. S.8 EDS mapping of (a) sample region of an unused particle of $\mathrm{Mg} / \mathrm{Zn}$ pictured by SEM elucidating distribution of (b) primary metal $\mathrm{Mg}$ to (c) oxygen, and (d) catalytic metal $\mathrm{Zn}$ 


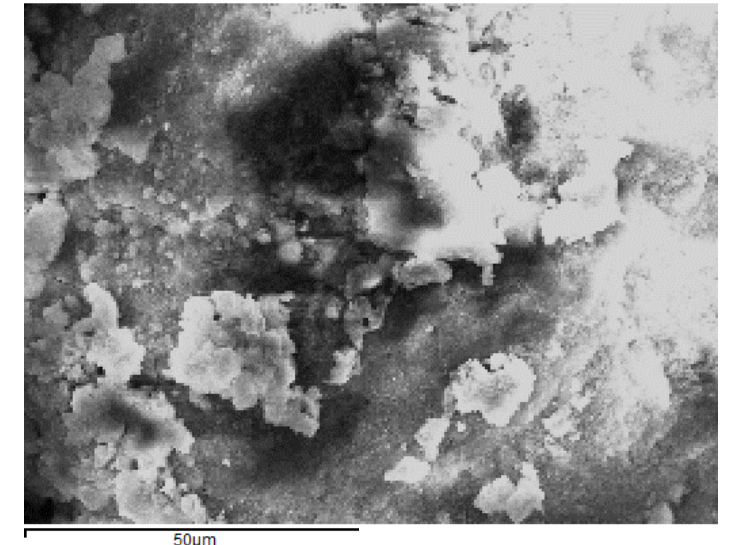

SEM: $\mathrm{Mg} / \mathrm{Zn}$

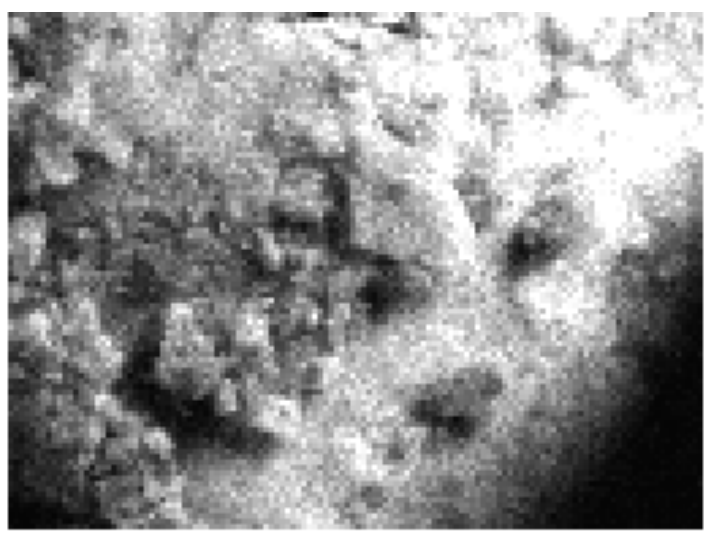

$\mathrm{O}$

(c)

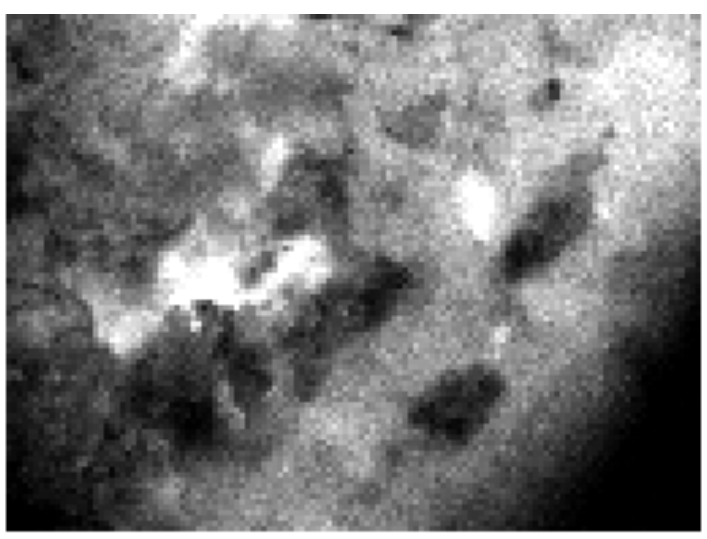

( b )

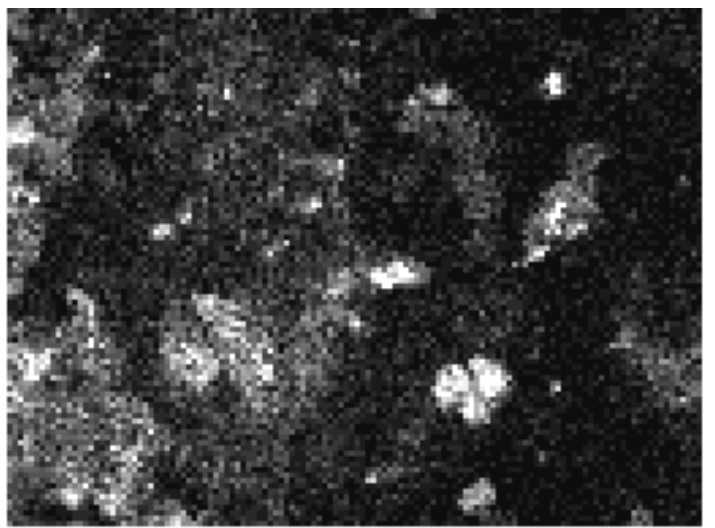

$\mathrm{Zn}$

(d)

Fig. S.9 EDS mapping of (a) sample region of a used particle of $\mathrm{Mg} / \mathrm{Zn}$ pictured by SEM elucidating distribution of (b) primary metal $\mathrm{Mg}$ to (c) oxygen, and (d) catalytic metal $\mathrm{Zn}$ 

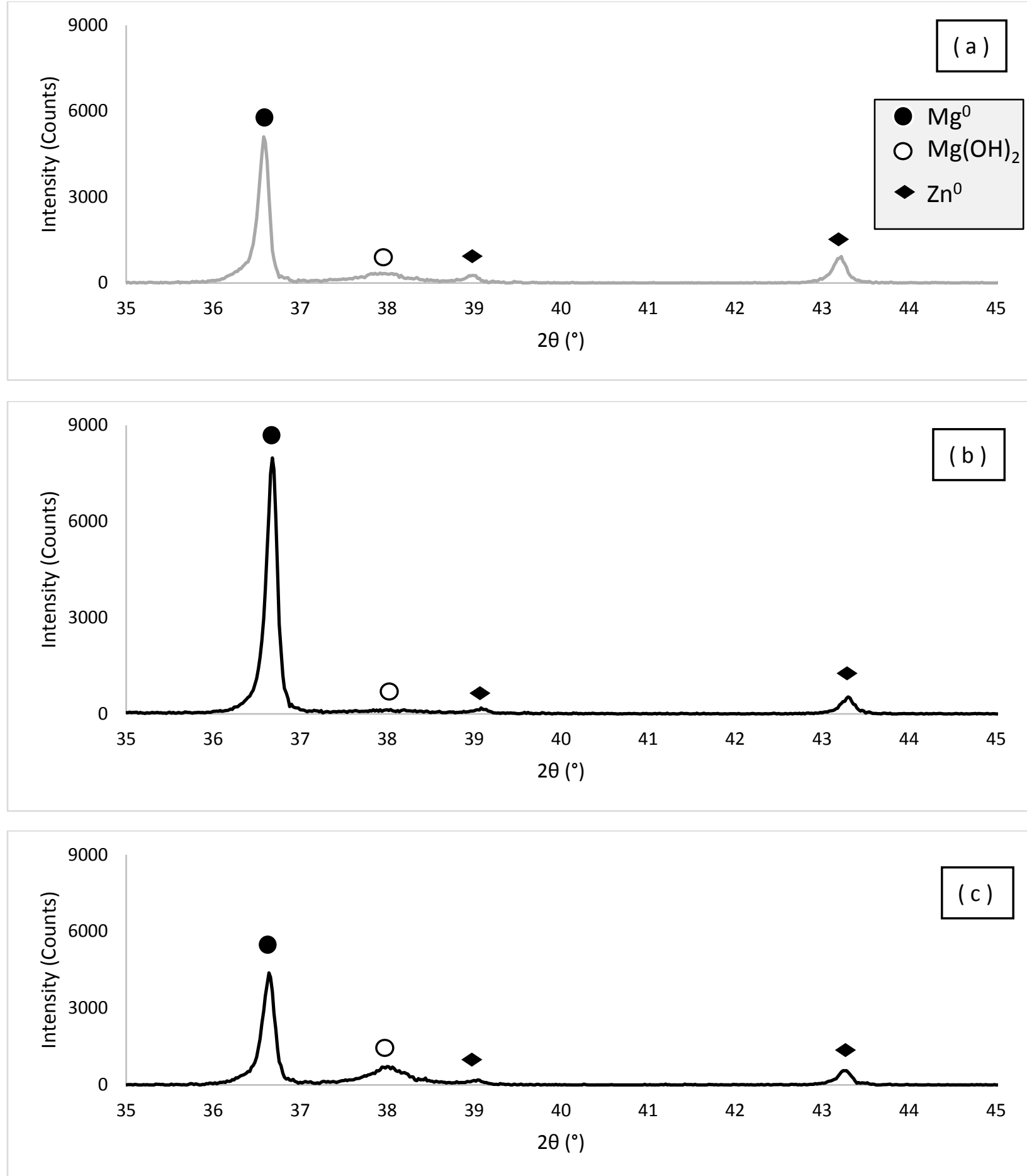

Fig. S.10 XRD patterns of $\mathrm{Mg} / \mathrm{Zn}$ (a) before treatment, (b) after treatment in wastewater, (c) and after treatment in the pure aqueous phase 


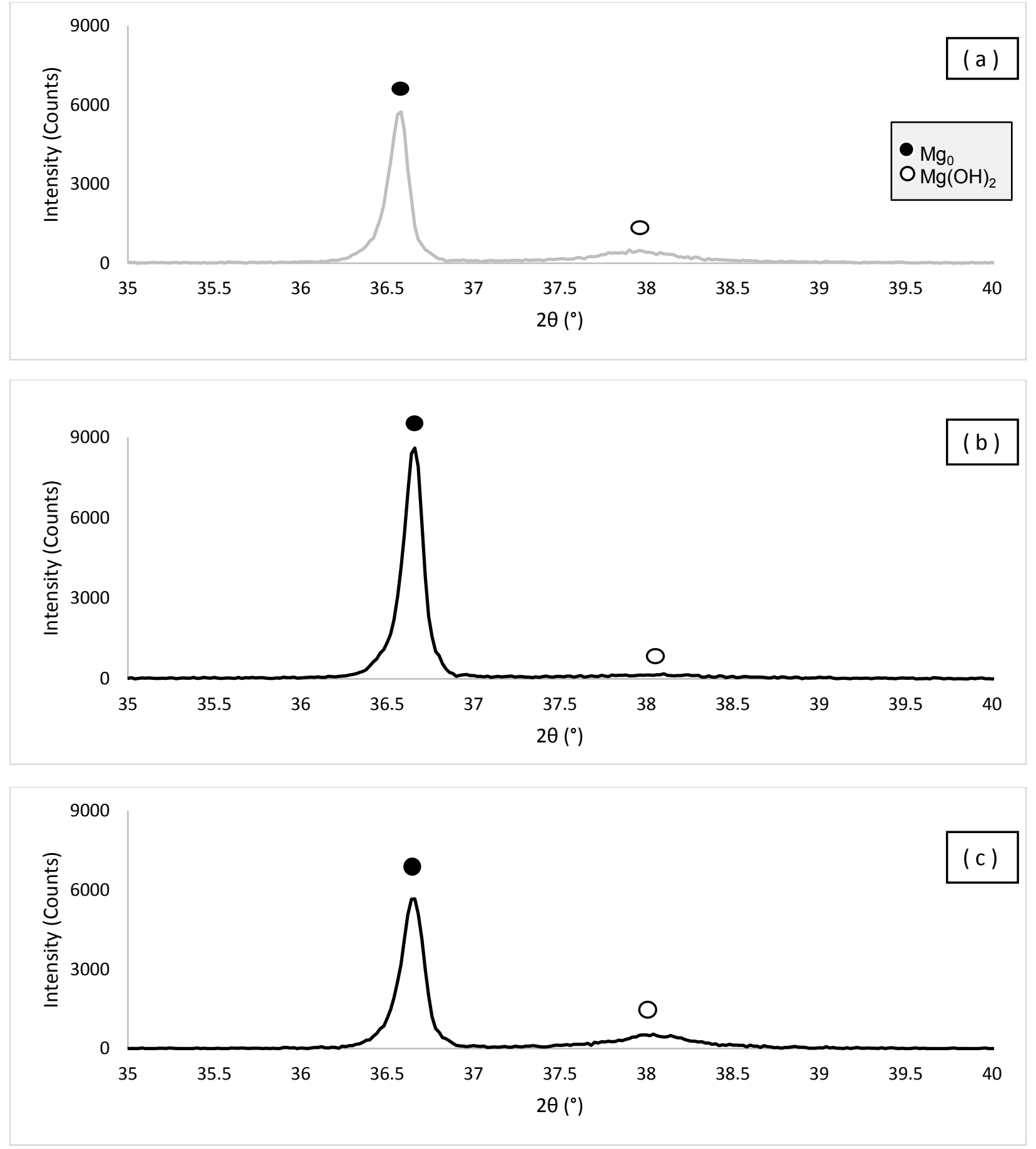

Fig. S.11 XRD patterns of $\mathrm{Mg} / \mathrm{Ni}$ (a) before treatment, (b) after treatment in wastewater, and (c) after treatment in the pure aqueous phase 